\title{
Elastic properties and multi-scale design of long carbon fiber nonwoven reinforced plane-based isotropic composite
}

\author{
Z. C. $\mathrm{He}^{\mathrm{a}}$, X. Shi ${ }^{\mathrm{a}}$, Eric $\mathrm{Li}^{\mathrm{b}}$, X.K. $\mathrm{Li}^{\mathrm{a}^{*}}$ \\ ${ }^{a}$ State Key Laboratory of Advanced Design and Manufacturing for Vehicle Body, Hunan University, Changsha, \\ 410082 P. R. China \\ ${ }^{\mathrm{b}}$ School of Computing, Engineering \& Digital Technologies, Teesside University, Middlesbrough, UK
}

\begin{abstract}
:
Carbon fiber reinforced composite is considered as a potential substitutes in the lightweight of automotive engineering. Nevertheless, the high price and complicated manufacturing process remain challenges for the large-scale application of conventional carbon fibers. In this study, a novel plane isotropic composite reinforced by long carbon fiber non-woven is introduced, and a multi-scale optimization method is proposed to realize the performance optimization and lightweight design of composite components. The linear elastic properties of long carbon fiber non-woven composite (LCFNC) were studied experimentally and analytically. The predictions of homogenization theory were similar to the experimental results within an interval of volume fraction. Then the relationship between microstructure and material performance was evaluated. The results showed that there is a critical point for the influence of aspect ratio to the material properties. The thickness of LCFNC at macro scales and the fiber parameters at micro scales were simultaneously optimized by Non-dominated sorting genetic algorithm-II (NSGA- II) to enforce multi-objective optimization design. Finally, the lightweight design of automobile hood was performed by the LCFNC and the proposed method. The results showed that the weight of automobile hood was reduced by $37 \%$, while exhibiting better stiffness and strength performance compared with the conventional steel.
\end{abstract}

Key words: Long carbon fiber nonwovens; Plane isotropic composite; Lightweight; Multi-scale optimization

\footnotetext{
*Corresponding author: Tel./fax: +86 73188822051.

Email address: ericsg2012@gmail.com (Eric Li); xkli8524@ sina.com (XK Li)
} 


\section{Introduction}

The demand for lightweight of vehicle is increasing due to the enactment of more stringent regulations of environmental pollution. In addition, the weight reduction of vehicle reduces running costs and fuel consumption, which can also satisfy the demand of fuel-efficient vehicle for consumers. The lightweight design of vehicle can be achieved by the extensively usage of vehicle structural optimization nowadays, and the move would be towards using more alternative materials, such as plastics and aluminum to help in weight reduction.

The use of lightweight materials is one of the most direct and effective means of achieving lightweight [1]. Compared with the metallic materials, the lightness and high specific strength of carbon fiber composite provides great opportunity to enhance structural performance and reduce the weight of automobile parts.[2-4]. Many automotive companies and carbon fiber producers have developed the carbon fiber composites to automotive parts, which enables vehicle body-weight reductions of an about 35-65 percent compared to competing materials. In 2015, BMW launched the 7 series of all-carbon body, which greatly reduced the weight of the body while improving the torsional stiffness of the cab. The i8 model also adopted a large number of high-strength and light-weight carbon fiber composites, making the weight of the vehicle less than 1.5 tons. In 2016, the roadster developed by KDX-Roding European R\&D Center used a carbon fiber composite body, which is lighter in weight and better in performance than traditional cars. However, due to its high cost and complicated process, carbon fiber composites are currently only employed in high-end cars and racing cars, and it is difficult to develop them on a large scale [5, 6]. Relatively speaking, the raw yarn of long carbon fiber non-woven can be extracted from the asphalt base, making the cost of it much cheaper than traditional carbon fibers. Besides, the rapid manufacturing of long carbon fiber 
non-woven is realizable, but its mechanical properties are relatively poor. The long carbon fiber non-woven provides a good balance between structural performance and cost. It is an ideal alternative for lightweight design of automotive parts with low performance requirements. Therefore, it is very meaningful and necessary to study the properties and applications of these novel plane isotropic composites.

Many scholars have systematically studied the mechanical properties of discontinuous fiber reinforced composite through theoretical, experimental and numerical simulation methods[7, 8]. The mean field inclusion method $[9,10]$ or the micromechanical method $[11,12]$ was adopted to predict the elastic modulus of discontinuous fiber reinforced composites. Hashimoto et al. [13] accurately predicted the tensile strength of discontinuous fiber reinforced composites by using multi-scale finite element method. Hartl et al.[14] studied the orientation dependence of tensile and compressive properties of short glass fiber reinforced polypropylene. Based on integrating up the orientation distribution function, Giordano[15] analyzed the effect of orientation on the elastic properties of composites by using Mori-Tanaka method. Berkache et al. [16-18] studied the mechanical properties of plane isotropic materials. In the present analytical models, the Mori-Tanaka (MT) mean field method and Eshelby's equivalent inclusion method have great utility to predict the mechanical properties of composites [19]. So far, researchers have conducted several studies on the properties prediction of fiber reinforced composite, but most of them focused on injection short fibers and woven composites, and there are very limited reports available on LCFNC. Moreover, the accuracy of model may be biased due to the different microstructures but few articles discuss the scope of the model.

On the other hand, structural optimization design is another method to achieve mass reduction of 
vehicle and has become very popular research topic among some scholars. Kc et al. [20] used taguchi method to optimize the molding parameters of fiber reinforced composites in order to reduce warping. Ghiasi et al. [21, 22] studied the optimization of lamination sequence of composites from the perspective of variable stiffness design and constant stiffness design. Blasques and Stolpe [23] achieved maximum stiffness and lightweight design by optimizing the angle and thickness of continuous fibers. Huang et al. [24] optimized the microstructure of the composite to maximize the stiffness of the output macrostructure. Belingardi et al. [25] found the best geometric parameters of the structure while meeting the design requirements. Based on the method of mathematical programming, Bruyneel [26] realized the optimization design of composite structures with thickness and direction as design variables. However, the above-mentioned optimization design of carbon fiber reinforced composites was mostly carried out unilaterally. The potential of mechanical properties and lightweight of materials and structures have not been fully exploited. How to fully consider the interaction between macro-structure design and micro-fiber parameters and establish a systematic two-scale concurrent optimization design model is still insufficient.

In this study, a novel plane isotropic composite was introduced, which makes it possible for carbon fiber to be widely employed in automotive parts with low strength requirements. In view of the shortcomings of the current research on LCFNC, the tensile properties of non-woven fabrics were predicted based on the homogenization theory and compared with the experimental results. In addition, the effects of key micro-parameters on properties of LCFNC were studied in detail and the scope of high-precision models was discussed. Then, on the basis of this novel plane isotropic composite, the coupling of macro-structure and micro-parameters was fully considered to realize the lightweight design of automotive hood. The finite element results showed that the hood met the basic 
performance requirements, while the hood mass was reduced by $37 \%$ compared to conventional steel. The current work provides technical guidance for LCFNC application in industry, and is of great significance for the process of promoting lightweight design.

\section{Characteristics of long carbon fiber nonwoven}

Due to the lightness and high specific strength, carbon fiber composites have obtained more focus in industry and academia. However, the high prices of these materials are serious obstacles to the large-scale application in manufacturing and the sophisticate fabrication processes and long production cycles (Fig.1) pose a huge challenge to mass production of traditional carbon fiber composites. In general, long carbon fiber non-woven has the advantages of simple process and preparation efficiency. As shown in Fig.1, the pitch-based are directly netted while being spun into fibers, and then carbonized or graphitized to form long carbon fiber non-woven fabrics after pre-oxidation. In addition, asphalt-based raw materials greatly reduce the cost of long carbon fiber nonwovens. The low cost and rapid manufacturing of long carbon fiber non-woven make it an ideal alternative to mass production of automotive parts[27].

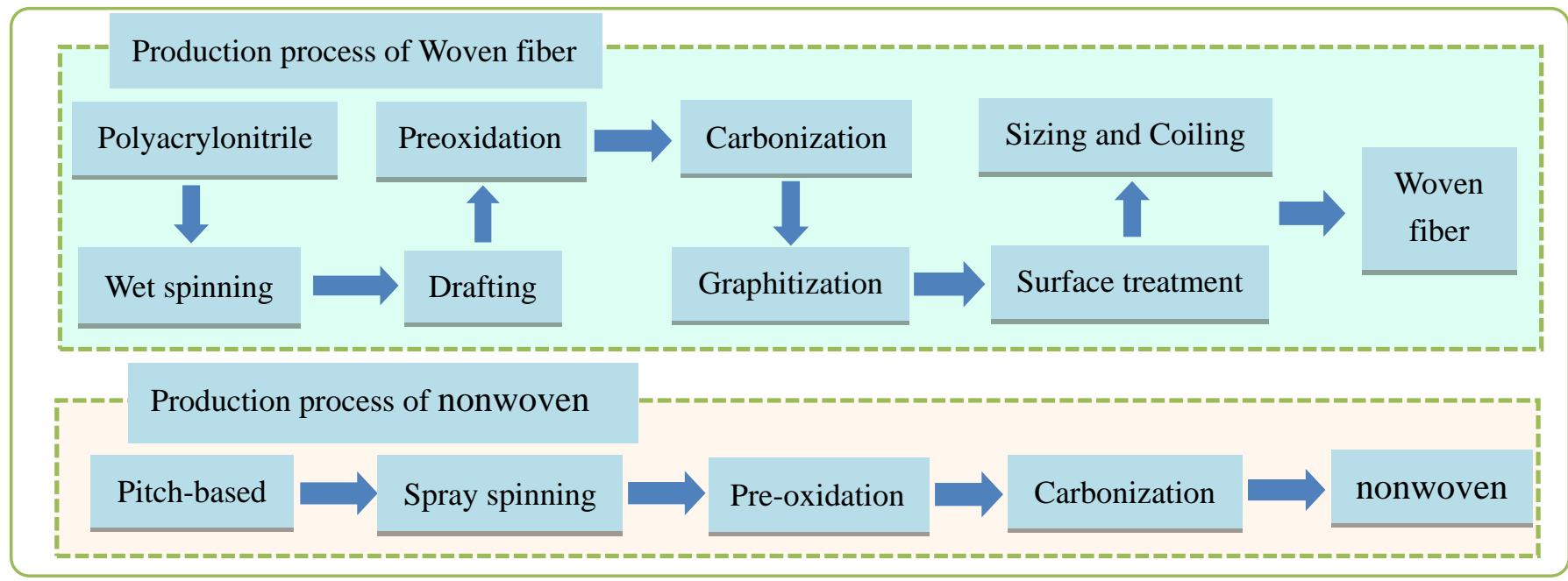

Fig. 1. Comparison of woven carbon fiber and non-woven molding process

Another characteristic of the long carbon fiber non-woven can be seen from the electron 
micrograph of Fig. 2. An electron micrograph of carbon fiber at 500um magnification is shown in Fig. 2 (a) and fibers with a certain curvature but approximately straight state are observed. In the appropriate volume fraction range, it can be seen from Fig. 2 (b) - (d) that different fibers are randomly crossed, and the overall distribution is disordered and uniform. This random fiber distribution in a two-dimensional plane results in a similar tensile strength of the LCFNC in all directions of the plane, which can be considered as a good in-plane isotropic composite. Besides, the fiber length of long carbon fiber non-woven is much longer than that of staple fibers, generally distributed in the $3-5 \mathrm{~cm}$ range.
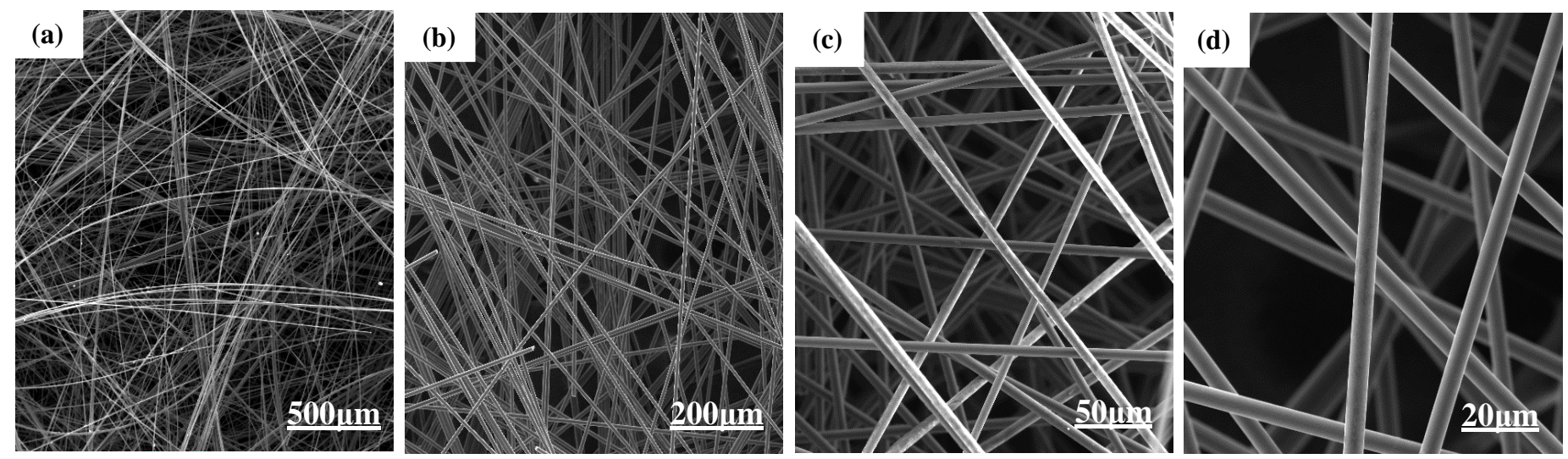

Fig. 2. Electron micrograph of different magnification of long carbon fiber non-woven

\subsection{Experiment and Analysis}

In order to quantitatively verify the plane isotropy of LCFNC, the tensile samples with different orientations of $0^{\circ}, 30^{\circ}, 60^{\circ}, 90^{\circ}$ (Fig. 3(a)) and different volume fractions were selected for tensile tests. 


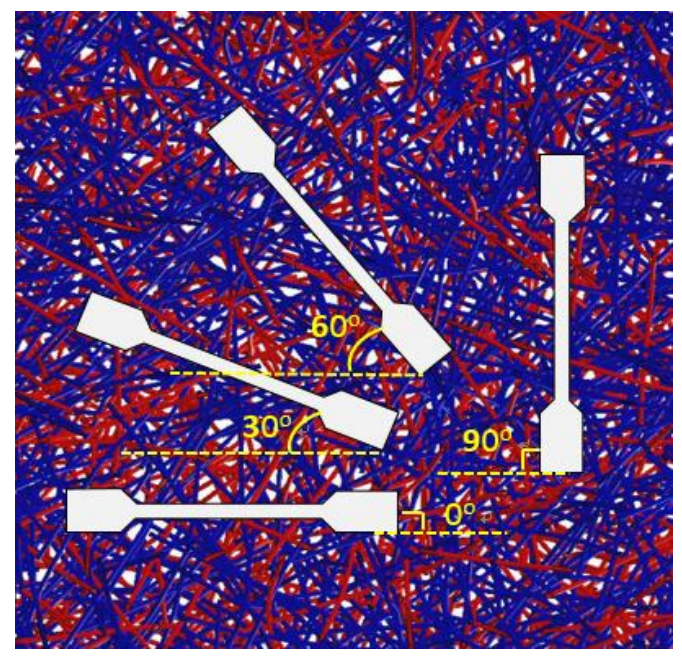

(a)



(b)

Fig. 3. Tensile specimens in different directions (a) schematic diagram; (b) experimental sample

Tensile test refers to the experimental method of measuring the material performance parameters under the axial tensile force. In this work, the tensile tests of LCFNC were conducted using an INSTRON-3382 electronic universal testing machine (Fig. 4(a)). The LCFNC specimens are $4 \mathrm{~mm}$ in thickness and $180 \mathrm{~mm}$ in length, and the specific size in details is shown in Fig. 4(c). The tensile modulus was measured by an extensometer (Fig. 4(b)) and the maximum stress in the test is considered as the tensile strength. The loading rate is set at $10 \mathrm{~mm} / \mathrm{min}$. For tensile test of LCFNC with different volume fraction, each test shall not be less than 5 groups of effective data, and the average values of mechanical properties shall be taken as the final test result. Tensile fracture was cut off for gold spray treatment, and the micro morphology of the fracture was observed by scanning electron microscope for subsequent analysis. 


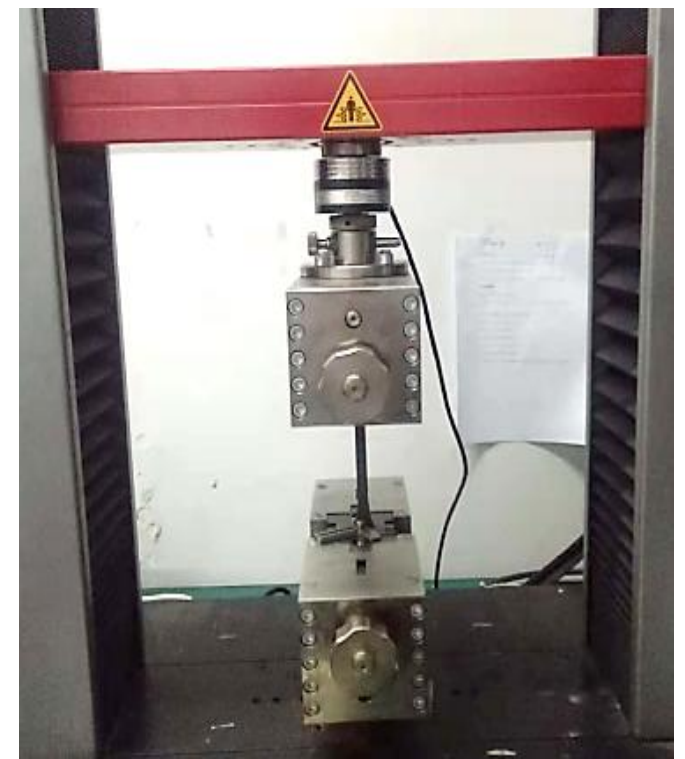

(a)

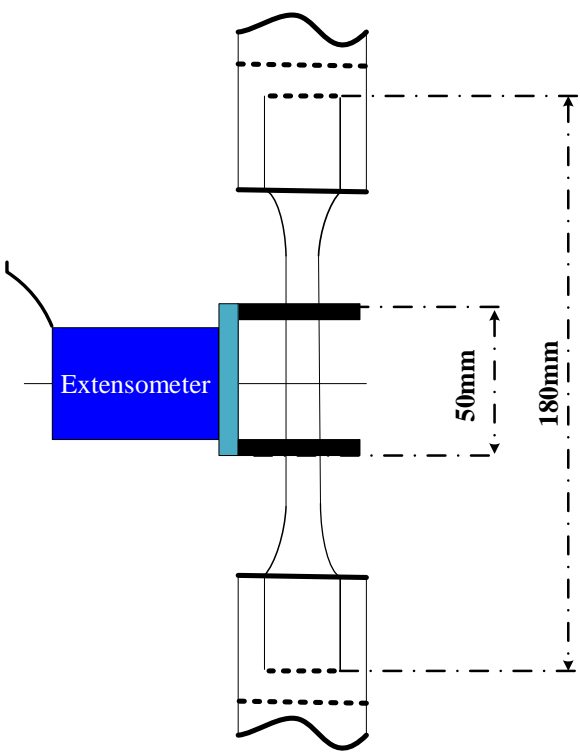

(b)

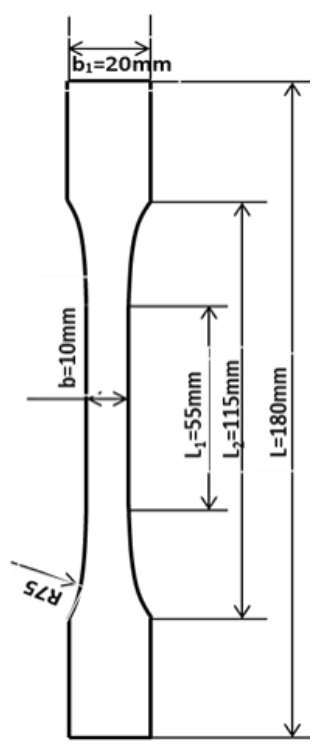

(c)

Fig.4.The uniaxial tensile test (a), the position of extensometer(b) and sample (c)

The tensile tests of composite samples with volume fraction of 0.2 and 0.3 were carried out, and the test results in different directions are shown in Figure 5 (a). The tensile strength of LCFNC in different directions is almost identical, which indicates that the material has a very good plane isotropic characteristic. It is worth noting that this isotropic material performance can only be shown when the volume fraction of the composite is appropriate. When the fiber content is lower or higher, the matrix-rich region or the fiber-dense region is prone to occur, resulting in a large difference in tensile strength, unstable mechanical properties, and anisotropic characteristics. Similarly, for composites with volume fractions of 0.1 and 0.5 , tensile specimens from different angles were selected for testing and the results were obtained as shown in Fig.5 (b). The tensile strength is random at different angles resulting in large performance deviation and tends to be anisotropic. 


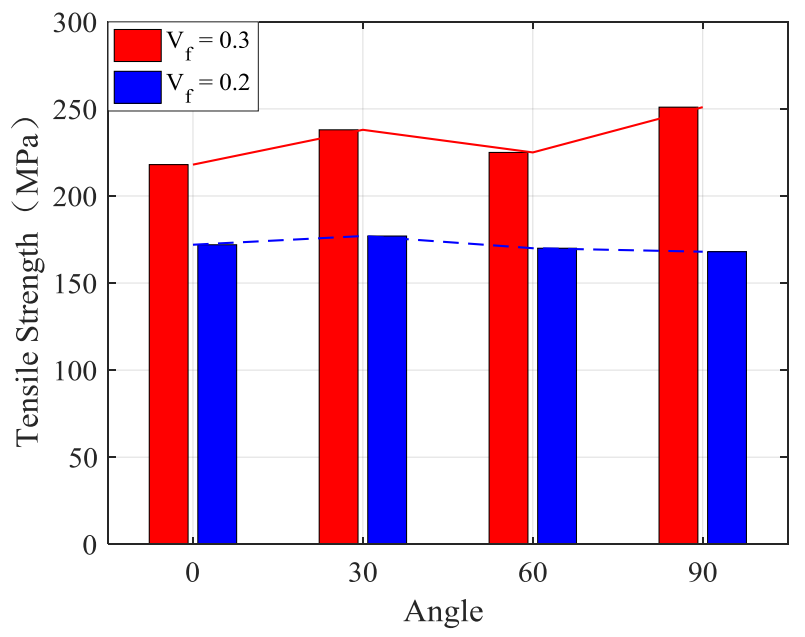

(a)



(b)

Fig. 5. Comparison of tensile strength of samples with different volume fractions in $0^{\circ}, 30^{\circ}, 60^{\circ}, 90^{\circ}$ directions

The fracture section of the LCFNC with different volume fractions are shown in Fig. 6. In Fig.6 (a)-(d), the fibers are sufficiently impregnated and the resin and fiber are better bonded. However, with the further increase of fiber content, fiber bundles begin to agglomerate, which makes it difficult for the resin to infiltrate. The electron microscopy (Fig.6 (e)-(f)) shows that the fiber and matrix are poorly bonded and the presence of local voids will lead to instability of composite properties. Based on experiment, it can be concluded that the properties of materials in different directions are si milar and composite reinforced by LCFNC has shown a very good plane isotropy when the volume fraction is in the range of $0.2-0.4$. 

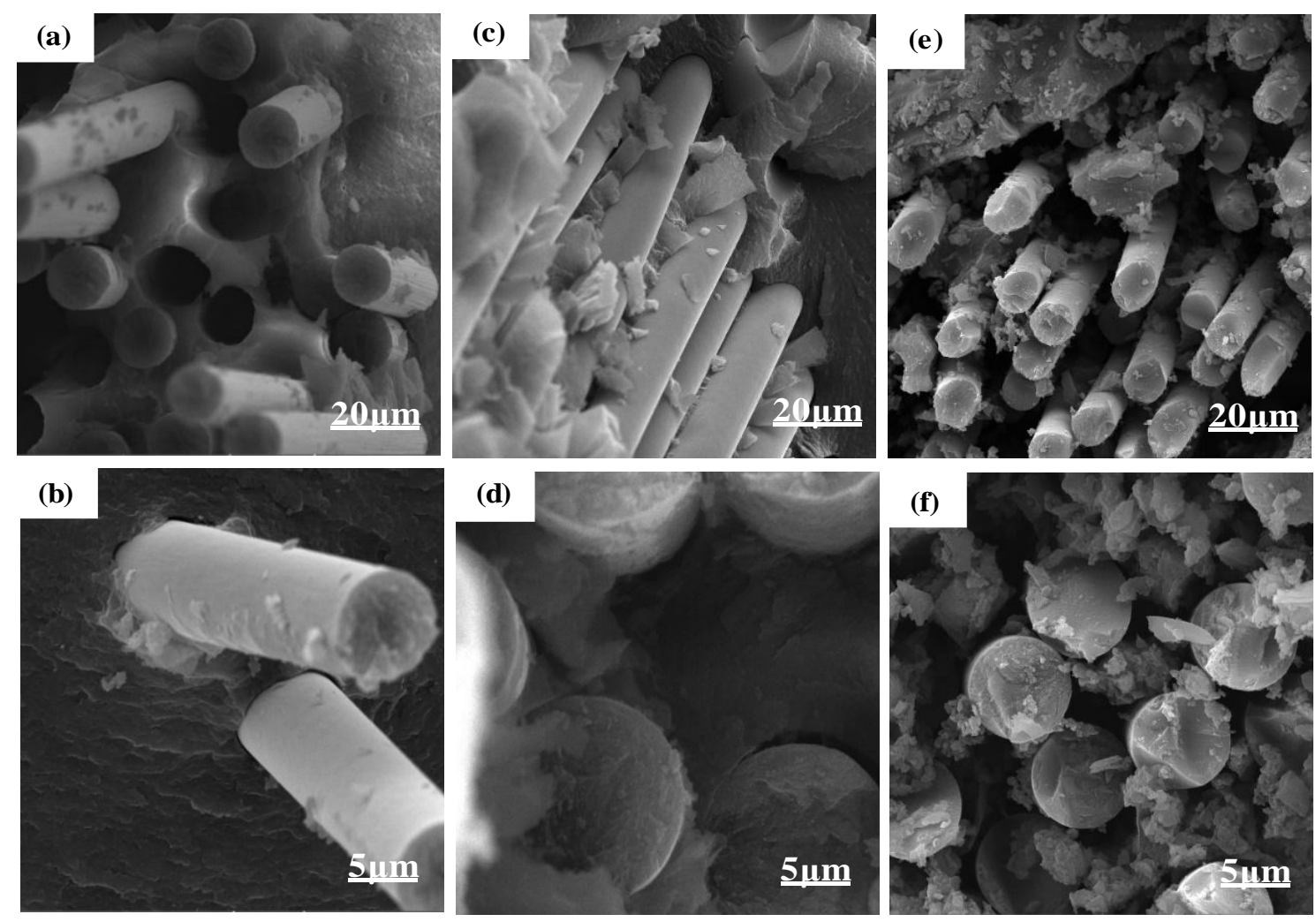

Fig. 6. Fracture surface of tensile specimens with different volume fractions and magnifications (a-b) 30\% volume fractions, (c-d) $40 \%$ volume fractions, (e-f) $50 \%$ volume fractions

According to the above analysis, composites molded by LCFNC show superior advantages in cost and process fabrication, compared with those molded by the traditional carbon fibers. LCFNC provides a good compromise between performance and cost, which is an ideal substitute for lightweight design of automotive parts. The transverse isotropy of long carbon fiber nonwoven fabric makes material have good mechanical properties in all directions of the plane, which enables the material to withstand complicated external force. In order to better realize the application and design of LCFNC, it is necessary to study and accurately express its performance.

\section{Simulations}

\subsection{Homogenization theory}

Homogenization is a procedure for calculating the elastic-plastic response of inhomogeneous materials by averaging the local field. Homogenization method is an effective method for analyzing 
the mechanical properties of fiber reinforced composites. It can predict the macro and micro mechanical properties of composites and guarantee an acceptable accuracy. The representative volume element (RVE), which is smaller than the structural size, but large enough to represent the microstructure [28] are firstly employed to describe the mechanical properties of LCFNC. By means of mean field homogenization (MFH) satisfying Hill-Mandel condition, the macroscopic performance parameters of RVE can be obtained by calculating the volume average of corresponding micro-quantities[29]. Different MFH methods will give different approximate descriptions of microscopic behavior. In this study, the Voigt and the Mori-Tanaka models[30], which are commonly used for discontinuous randomly oriented fiber-reinforced composites, are adopted to analyze the properties of materials.

In the modelling of a $\operatorname{RVE} \varphi$ with two-phase composite materials, which consists of the matrix $x_{0}$ (phase 0) with the volume fraction of $v_{0}$ and the inclusions $x_{1}$ (phase 1) having a volume fraction of $v_{1}$, the relationship between the average value of the RVE, the matrix phase, and the fiber phase can be expressed as follows by the rule of homogenization method.

$$
\langle f\rangle=v_{0}\left\langle f_{0}\right\rangle+v_{1}\left\langle f_{1}\right\rangle=\left(1-v_{1}\right)\left\langle f_{0}\right\rangle+v_{1}\left\langle f_{1}\right\rangle
$$

where $f$ represents micro-stress/strain field on RVE, subscript ' 0 ' and ' 1 ' are the matrix and inclusion property, respectively;

Under the boundary condition of linear displacement, the relationship between macro strain and the average strain of per phase can be expressed by strain concentration tensor $\mathbf{T}$ [31].

$$
\begin{aligned}
& \langle\varepsilon\rangle_{\varphi 1}=\mathbf{T}:\langle\varepsilon\rangle_{\varphi 0} \\
& \langle\varepsilon\rangle_{\varphi 0}=\left[v_{1} \mathbf{T}+\left(1-v_{1}\right) \mathbf{I}\right]^{-1}:\langle\varepsilon\rangle \\
& \langle\varepsilon\rangle_{\varphi 1}=\mathbf{T}:\left[v_{1} \mathbf{T}+\left(1-v_{1}\right) \mathbf{I}\right]^{-1}:\langle\varepsilon\rangle
\end{aligned}
$$

where I designates the $4^{\text {th }}$ order symmetric identity tensor. For different homogenization models, 
tensor $\mathbf{T}$ has a different description, but the equivalent stiffness matrix of the composite material can always be written as:

$$
\langle\mathbf{C}\rangle=\left[v_{1} \mathbf{C}_{1}: \mathbf{T}+\left(1-v_{1}\right) \mathbf{C}_{0}\right]:\left[v_{1} \mathbf{T}+\left(1-v_{1}\right) \mathbf{I}\right]^{-1}
$$

where $\mathbf{C}_{\mathbf{1}}$ is uniform stiffness of inclusion and $\mathbf{C}_{0}$ is uniform stiffness of matrix.

The Mori-Tanaka model [31] relates the strain of inclusions to the uniform strain of the far field action [32];

$$
\varepsilon(x)=\mathbf{H}^{\varepsilon}\left(\mathbf{I}, \mathbf{C}_{0}, \mathbf{C}_{1}\right): \bar{\varepsilon} \quad \forall x \in \varphi_{1}
$$

$\mathbf{H}^{\varepsilon}$ is the fiber strain tensor can be expressed as,

$$
\mathbf{H}^{\varepsilon}\left(\mathbf{I}, \mathbf{C}_{0}, \mathbf{C}_{1}\right)=\left\{\mathbf{I}+\mathbf{S}:\left[\mathbf{C}_{0}^{-1}: \mathbf{C}_{1}-\mathbf{I}\right]\right\}^{-1}
$$

where $\mathbf{S}$ represents Eshelby's tensor. For the $\mathbf{M}-\mathbf{T}$ model $[10,19]$ the tensor $\mathbf{T}$ is equal to the fiber strain tensor

$$
\mathbf{T}=\mathbf{H}^{\varepsilon}\left(\mathbf{I}, \mathbf{C}_{0}, \mathbf{C}_{1}\right)
$$

Substituting Eqs. (5) and (6) into Eq. (3), the equivalent stiffness matrix $\mathbf{C}$ can be obtained.

In the Voigt model, it is based on the assumptions that the strain of the composite is equal to the average strain of the single-phase material [33]

$$
\langle\varepsilon\rangle_{\varphi}=\left(1-v_{1}\right)\langle\varepsilon\rangle_{\varphi_{0}}+v_{1}\langle\varepsilon\rangle_{\varphi_{1}}=\langle\varepsilon\rangle_{\varphi_{0}}=\langle\varepsilon\rangle_{\varphi_{1}}
$$

Substituting Eq. (7) into Eq.(2), it can find $\mathbf{T}=\mathbf{I}$, and then the effective stiffness tensor of the composite can be expressed as

$$
\langle\mathbf{C}\rangle=v_{1} \mathbf{C}_{1}+\left(1-v_{1}\right) \mathbf{C}_{0}
$$

LCFNC consists of a matrix material and carbon fiber filaments. The purpose of MFH is to find an equivalent composite whose elastic modulus and the relationship of stress-strain are the same as the composite in a certain direction. The mechanical properties of LCFNC can be replaced by this 
homogeneous material. By establishing a mesoscopic fiber homogenization RVE model, the macroscopic mechanical response of the fiber reinforced composite can be effectively predicted.

\subsection{Component properties and micro models}

In this paper, the matrix material which has excellent heat resistance and corrosion resistance is composed of vinyl resin and unsaturated epoxy resin. We made the matrix material into a dumbbell shape for uniaxial tensile test. Since the tensile strain at break of the matrix material far exceeds the composite, the maximum strain of the substrate is set at $4 \%$. Based on the J2-plastic model [34] , the experimental results of the stress-strain curve of the matrix were fitted and the model parameters were used in the simulation analysis of the composite.

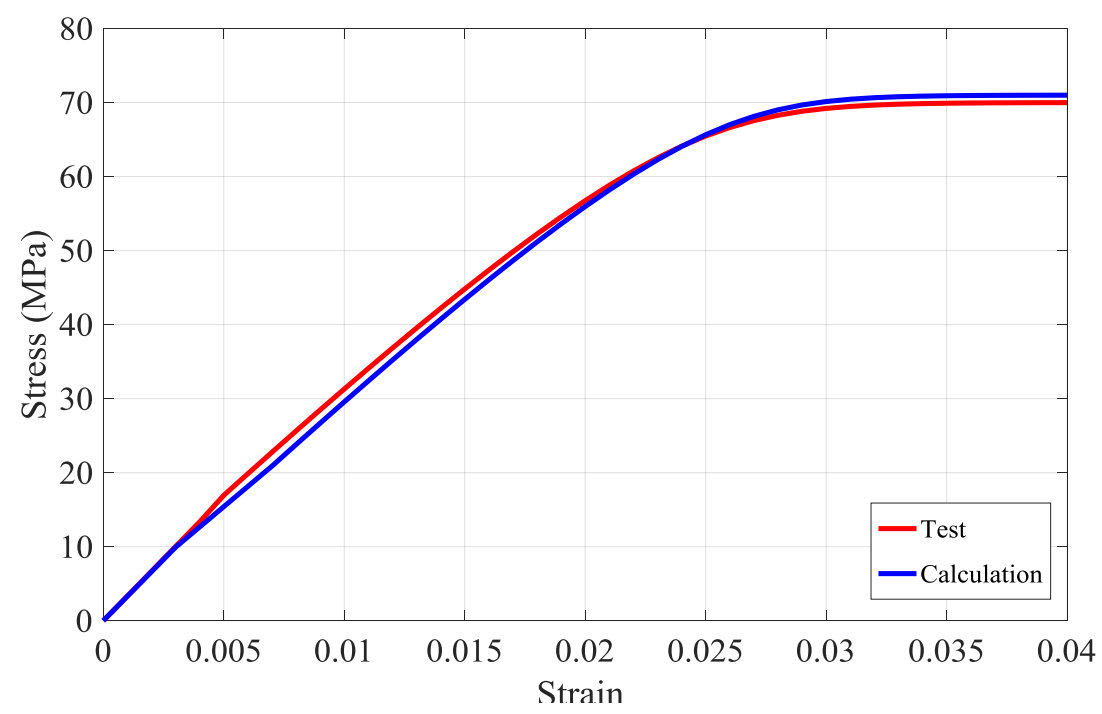

Fig. 7 Comparison of stress-strain curves of matrix materials

The stress-strain response of the matrix is shown in Fig 7 and the experimental curve fits well with the curve calculated based on J2-plastic model. When the stress exceeds the threshold of yield stress, the material shows nonlinear behavior. Carbon fiber t700 was used as the inclusion to improve the mechanical properties of composites. The performance parameters of carbon fiber and the model parameters for fitting stress-strain curves of matrix materials were obtained as shown in Table 1. 
Table 1 performance parameters for numerical computations

Young's Modulus (GPa) Poission's ratio Density $\left(\mathrm{g} / \mathrm{cm}^{2}\right) \quad$ Length $(\mathrm{mm})$

Elongation (\%)

Inclusion

230

0.31

1.8

$30-50$

2.0

Young's Modulus (GPa) Poission's ratio Density $\left(\mathrm{g} / \mathrm{cm}^{2}\right) \quad$ Yield stress(MPa) Harding modulus (MPa)
Matrix
3.34
0.4
1.1
16
54

Because the compression process of composites causes fiber overflow, the $V_{f}$ of LCFNC is measured after molding. The actual $V_{f}$ of the composite is determined by measuring the overall $V$ of the composite and the weight of $M_{c}$ by burning the resin. $\rho_{c}$ is the density of non-woven carbon fiber used in the material. The following equation is employed to calculate:

$$
V_{f}=\frac{M_{c} / \rho_{c}}{V}
$$

At the same time, we also determined the aspect ratio of carbon fiber filaments. For carbon fiber nonwovens, 500 units were obtained, and the length distribution was statistically calculated. The aspect ratio can be obtained by the following simple equation:

$$
F_{c}=\frac{l}{d}
$$

where $F_{c}$ represents the aspect ratio of carbon fiber, $l$ and $d$ are the fiber length and diameter, respectively. In this study, periodic boundary conditions are used for the RVE analysis, which is implemented by a series of constraint equations to connect the nodes on a plane with the corresponding nodes on the opposite plane. The displacement field $u$ is described as follows [35]:

$$
u(x+L)=u(x)+\varepsilon \bullet x \quad \forall x \in \partial V
$$

where $\varepsilon$ denotes the strain field, $L$ is the dimension of RVE. By integrating the node displacement distribution, the average displacement of RVE can be obtained. 
The modelling of periodic RVEs reinforced by the inclusions is conducted to predict the effective elastic constants of LCFNC. The properties of the composite are directly related to the microscopic parameters. In the process of modeling, the model parameters such as volume fraction, fiber orientation and aspect ratio, should be set to fit the real microstructure. Two-layer RVE models with different volume fractions and their corresponding inclusions are shown in Fig. 8. The size of RVE is $L \times L \times t$, and its specific size is calculated automatically by the finite element software according to the microstructure definition of LCFNC. The portion of the resulting fiber that transcends the RVE boundary will be cut and transferred to the respective opposite sides, which is prerequisite for the RVEs to apply periodic boundary condition analysis. The orientation of the fibers is set to Random 2D, which is randomly oriented in the plane to ensure the plane isotropy of the material. In order to show the distribution of fibers clearly, the matrix of RVEs was blurred. The blue cylinder represents the first layer of fibers and the second layer of fibers is red, which is uniformly distributed as a whole. Uniaxial strain loading is applied to the RVE boundary and the initial strain and peak strain are set to 0 and 0.03 respectively. The analysis terminates when the peak value is reached. The structural components and data measured in these analyses were adopted for simulation based on Mori-Tanaka model and Voigt model. The multi-scale homogenization software Digimat was used for calculation in this study.
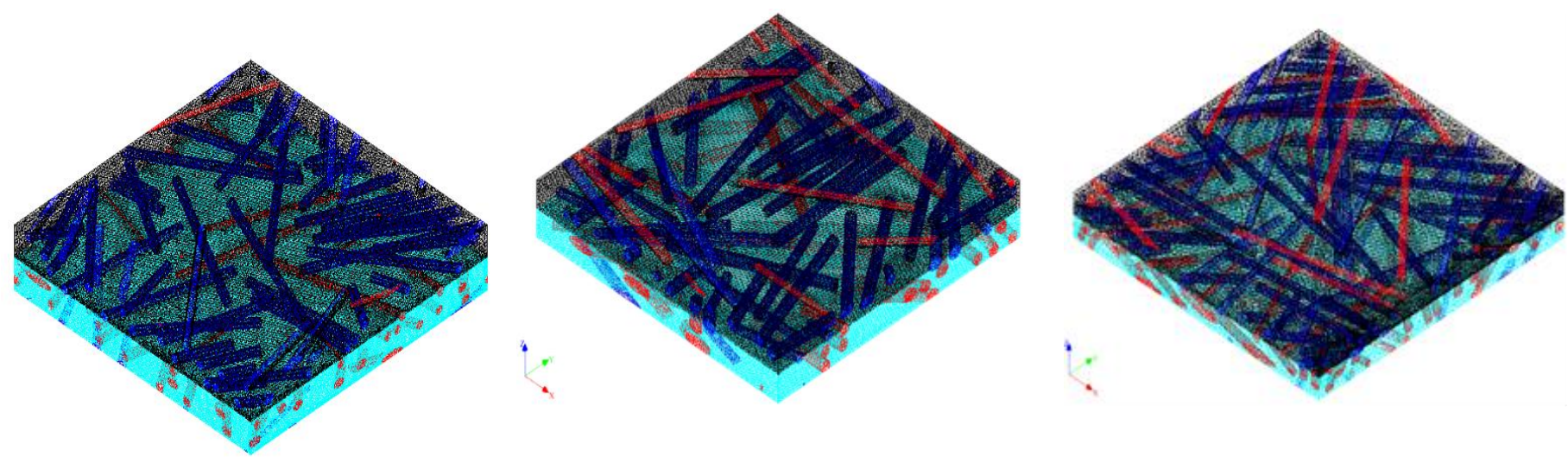
Fig.8. RVE model with different volume fractions.

(a) $20 \%$ volume fraction, (b) $25 \%$ volume fraction, (c) $30 \%$ volume fraction

\section{3 result and comparison}

Similarly, tensile tests were carried out on the composites with the corresponding volume fraction to verify the accuracy of the homogenization model. The stress-strain curves obtained from the experimental and simulation results are shown in Fig. 9. Two sets of data of LCFNC with volume fractions of $20 \%$ and $30 \%$ were compared. The results indicated that simulated stress-strain curves show considerable agreement with those generated from the experiments and both of them show good linearity. The main difference is that the simulation curve is generally higher than the experimental curve.

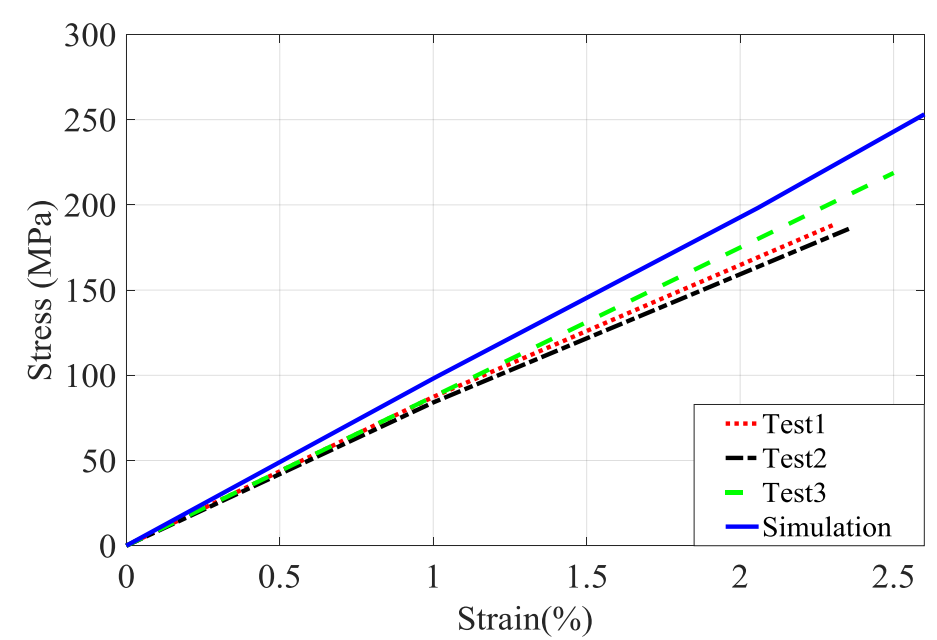

(a)

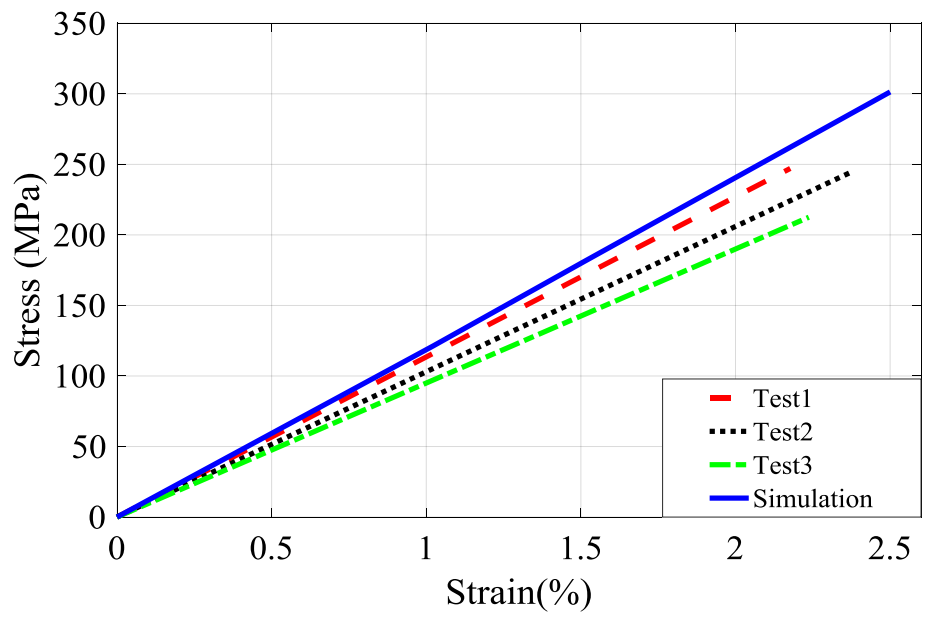

(b)

Fig.9. Stress-strain curves of the experiment and simulation results with different volume fraction: (a) $20 \%$; (b) $30 \%$.

To further verify the reliability of simulation results based on homogenization method, the experimental and simulation results of the in-plane Young's modulus and maximum tensile strength before the final rupture occurs were compared, as shown in Fig.10. The simulation results and experimental results show a good consistency overall within the range of 0.2 to 0.4 volume fraction. There are some deviations that the simulation results are usually slightly higher than the 
experimental values, but the deviations are within acceptable error range. Besides, the Young's modulus and tensile strength increase with the increase of fiber volume fraction, which is typical for this novel plane isotropic composite because the performance of carbon fiber is much higher than that of the matrix. It is worth noting that when the volume fraction exceeds 0.4 , the experimental results of tensile strength and Young's modulus begin to decline. However, the results of the simulation prediction continue to rise, and the deviation between the simulation and the experimental results increases. This anomaly is mainly due to the difficulty of impregnation of fibers at high volume fractions, which is easy to form voids. Besides, the fiber is prone to uneven distribution under the condition of low volume fraction. These abnormal states make the properties of LCFNC tend to be unstable and weakened, making it difficult to predict.

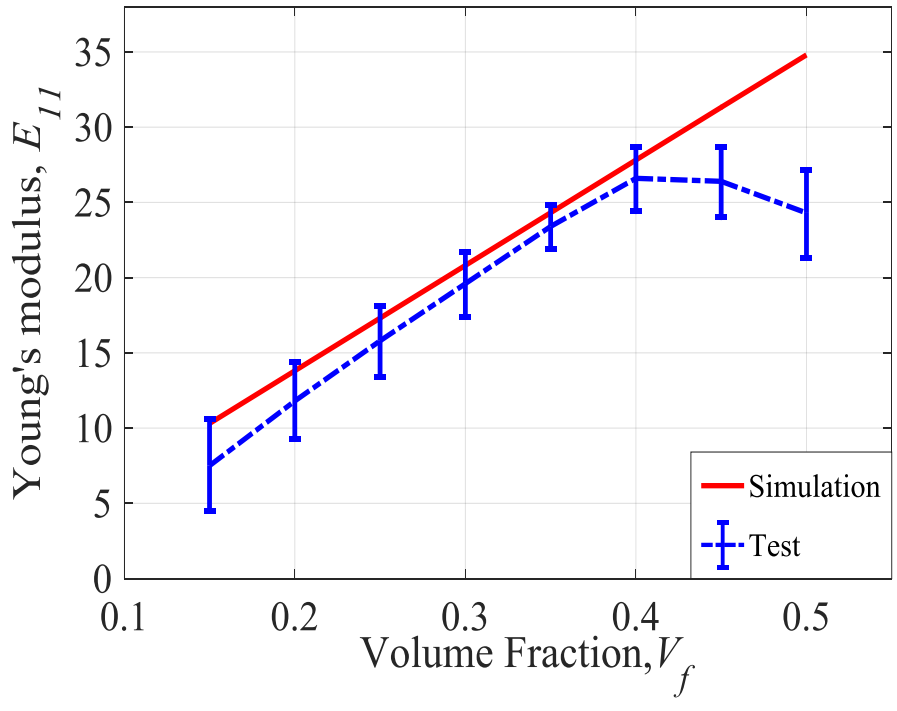

(a)

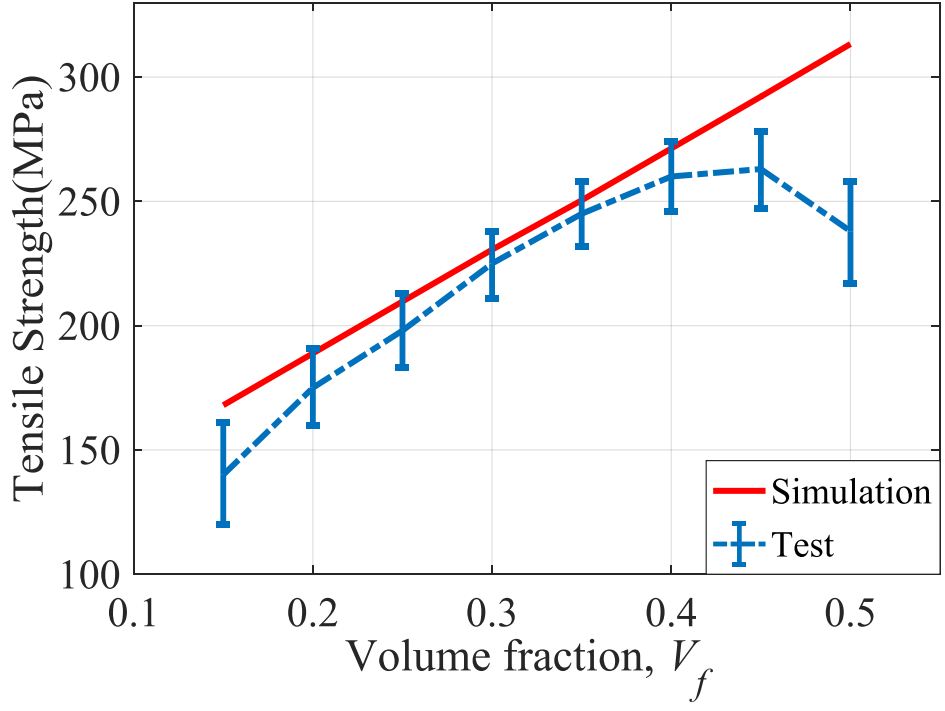

(b)

Fig.10. Comparison of mechanical properties with different volume fractions between test and simulation,

(a) Young's modulus, (b)Tensile strength

Based on numerical simulation and experiment, it can be concluded that the elastic properties of LCFNC obtained by the homogenization analysis method can be well matched with the experimental results and the model has shown very good accuracy when the volume fraction is between 0.2 and 0.4. It also indicates the homogenization method is capable to predict the elastic properties of LCFNC. 
Therefore, it is feasible to apply the elastic parameters of LCFNC predicted from the microscopic scale to mesoscale analysis.

\subsection{Microscopic parameters and mechanical properties}

It has been found that the microstructure of composite affects the mechanical properties of fiber-reinforced composites [36-38]. In order to better realize the optimal design and application of LCFNC, it is necessary to quantitatively analyze the microscopic parameters that affect the elastic constant. According to the above analysis, the fiber homogenization of composite with different components was carried out, and the influence of fiber aspect ratio and fiber content on the mechanical properties of LCFNC was studied in detail based on the simulation results.

The simulation based on the homogenization model was conducted by using of periodic boundary conditions and applying 3\% peak strain to the RVE. Within the valid range of the model, the influence of fiber aspect ratio on the mechanical properties of composites was analyzed by keeping the fiber content constant. The results have been obtained and shown in Fig. $11(a-b)$. The subscript 1 represents in-plane performance parameters and subscript 2 represents out-of-plane performance parameters. The following is the same unless otherwise specified.

Both the $E_{1}$ (in-plane Young's modulus) and $G_{1}$ (in-plane shear modulus) improve with increasing aspect ratio. Specifically, $E_{1}$ and $G_{1}$ show the same trend that they are shown as quadratic functions of variable when the aspect ratio of the fibers is low, and then gradually become stable. One thing worth noting is that the aspect ratio of the fiber has a critical value for the gain of the in-plane performance parameter of the LCFNC, and this critical point hardly changes for different volume fraction composites. When the aspect ratio of the fiber exceeds 200 , the in-plane properties of the composite will not change as the aspect ratio increases further. It can also be seen that the 
aspect ratio of the composite has little effect on the out-of-plane performance parameters.

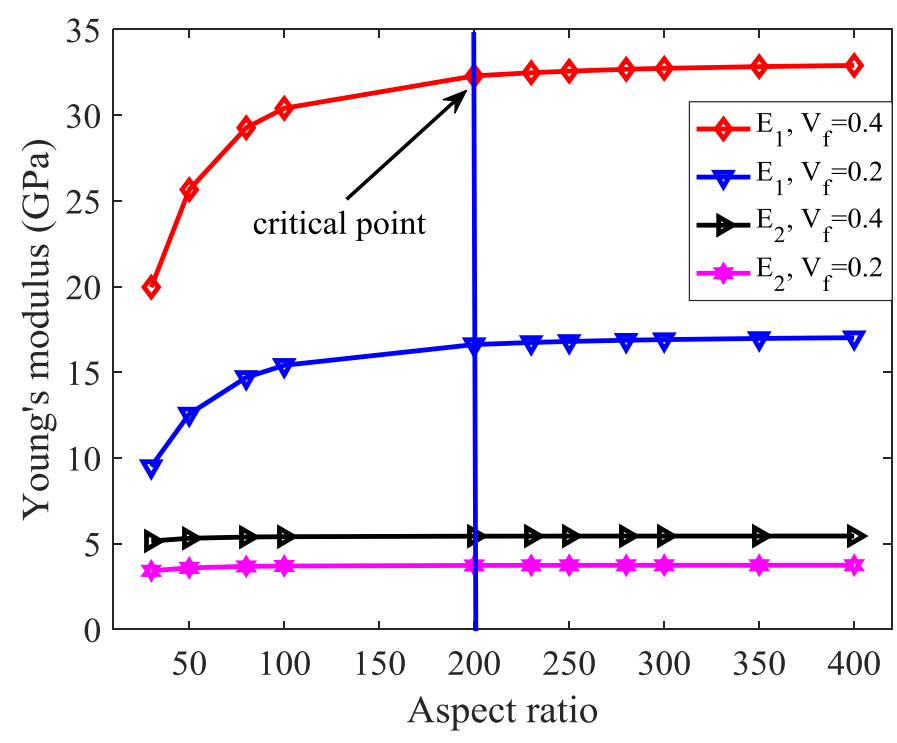

(a)

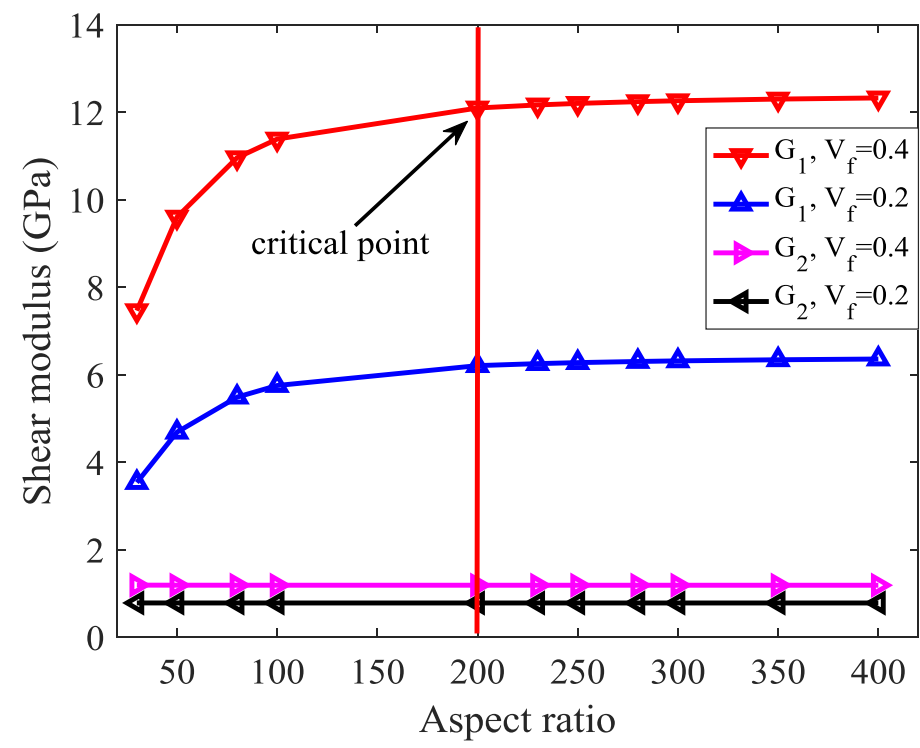

(b)

Fig. 11. Effects of fiber aspect ratio on the Young's modulus (a), and shear modulus (b)

In order to study the effect of volume fraction on the out of plane effective modulus and Poisson's ratio of LCFNC, the simulation with the same aspect ratio and different values of volume fraction were performed. The results are shown in Fig. 12. The relationship between the out-of-plane performance parameters and volume fraction is the same as that of the plane (Fig. 10). It can be concluded that the tensile and shear stiffness of composites in all directions can be improved by increasing the volume fraction. Regarding the Poisson's ratio, with the increase of volume fraction, $u_{2}$ (out-of-plane Poisson's ratio) decreases gradually and then tends to be stable. However, the variation tendency of $u_{l}$ (in-plane Poisson's ratio) is almost not affected by the change of volume fraction. Through Fig.10 and Fig.12, it is found that the tensile properties of in-plane are much higher than those of out-of-plane and the difference between in-plane Young's modulus and out-of-plane Young's modulus increases with the increase of volume fraction. 


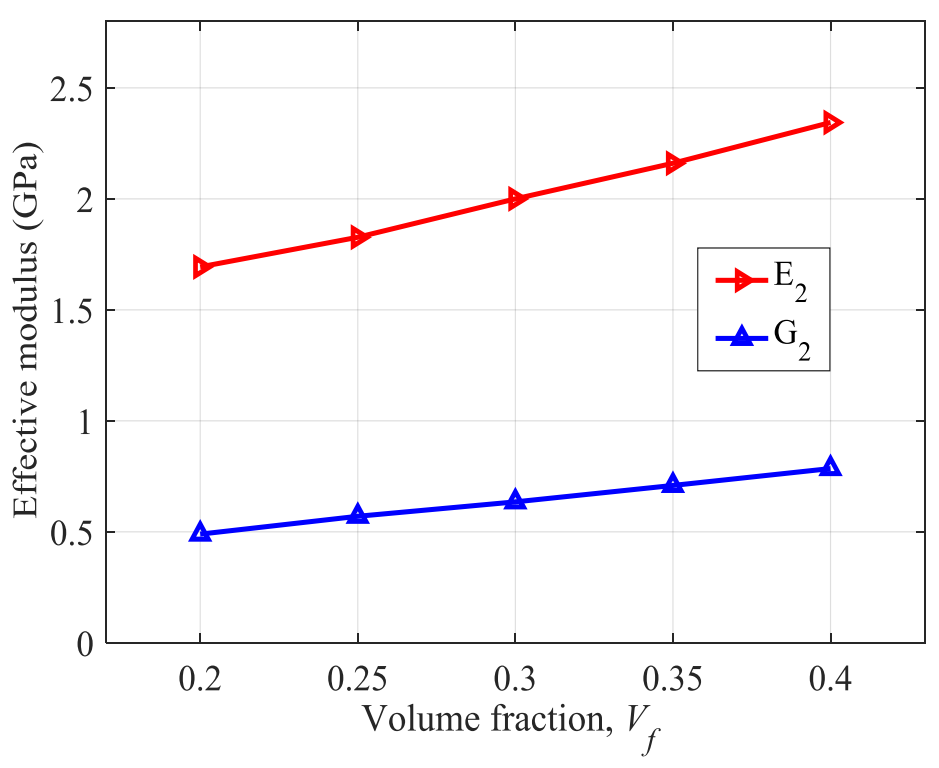

(a)

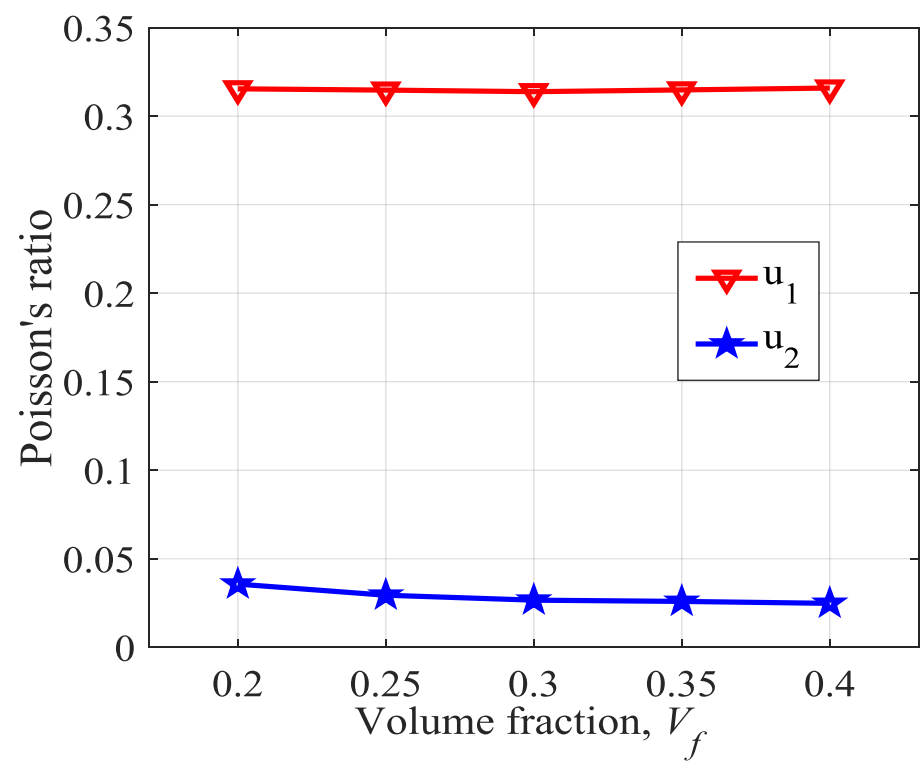

(b) Poisson's ratio

Fig. 12. Effects of volume fraction on the Effective Moduli (a), and Poisson's Ratio (b)

\section{Multi-scale optimization design of composite}

The structural optimization can fully exploit material properties while reducing costs, which plays an increasingly important role in the design process of components. The ideal design of a component should satisfy both the optimum macro-topological optimization and the optimum micro-parameters. Therefore, the coupling of macro-structure and microscopic parameters should be fully considered in the optimization design.

On the basis of theoretical study of material properties, the LCFNC was applied to the optimization design of automobile parts, and multi-objective optimization algorithm was employed to achieve lightweight while maximizing stiffness. For different composite auto parts, the macroscopic structure of components and the microscopic parameters of single-phase materials ultimately determine the overall performance of components. The multi-scale and multi-objective optimization design can be achieved as shown in Fig. 13. 

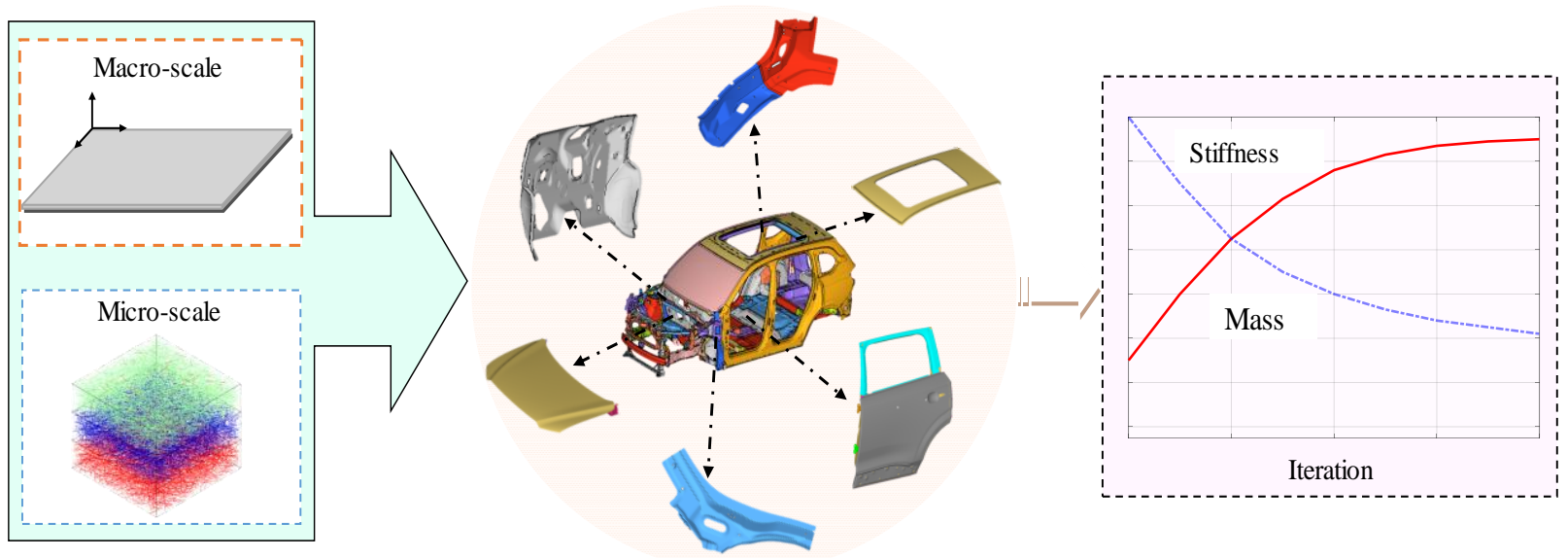

Fig.13. Multi-target and multi-scale design of automotive parts

For different parameters study, the reasonable selection of sampling points through Design of Experimental (DOE) method is fundamental to ensure the ultimate optimization effect and efficient implementation of the optimization process. Among many DOE, Optimal Latin hypercube sampling (OLHS) is often adopted to study multi-factor problems [39, 40]. It can generate sampling points randomly and evenly while maintaining high efficiency. Besides, Non-dominated sorting genetic algorithm (NSGA-II)[41] was applied to perform deterministic optimization in this study. NSGA-II is a genetic algorithm for multi-objective optimization in a non-dominated form, which is efficient and fast, and the Pareto non-inferior solution is closer to the ideal frontier. At present, NSGA-II algorithm has been widely adopted in many field[42, 43], and has achieved good optimization results.

In this study, the thickness of components and the volume fraction of LCFNC were adopted as design variables and the objective of optimization is to reduce the mass of components and improve the lateral stiffness on the premise of guaranteeing the basic performance requirements. Stiffness and modal are the main performance indexes of automotive parts and it should be controlled within the corresponding target value. The final optimization equation of multi-scale and multi-objective are obtained as follows: 


$$
\int \begin{array}{r}
\text { Find }: h_{i} \text { on macro-scale } \\
V_{f} \text { on micro-scale }
\end{array}
$$

Min: $m, u_{1}$

$$
\left\{\begin{array}{c}
\text { s.t.: } c_{1}-N_{1} \leq 0 \\
u_{2}-N_{2} \leq 0 \\
\kappa-R \geq 0 \\
\text { where } V_{f} \in\left(V^{l}, V^{u}\right) \\
h_{i} \in\left(h^{l}, h^{u}\right), i=1,2, \ldots, n
\end{array}\right.
$$

where $m$ is the total mass of the component and $u_{1}$ is displacement of the loading point during lateral stiffness analysis. $c_{1}$ is torsional stiffness. $\boldsymbol{\kappa}$ is the first order mode. $u_{2}$ is the displacement of the loading point during bending stiffness analysis. $V_{f}$ is the volume fraction of the composite. $V^{l}$ and $V^{u}$ are the upper and lower bounds of the volume fraction, respectively. $h_{i}$ is the thickness of parts. $h^{l}$ and $h^{u}$ are the upper and lower bounds of thickness, respectively. $N_{1}, N_{2}, R$ are the critical value of performance requirements for each working condition. The $n$ is the number of optimized parts. In this work, the automobile hood is taken as the study object. From the micro-structure to macro-scale, combined with the optimal Latin hypercube sampling (OLHS) and non-dominant sequencing genetic algorithm-II, multi-objective optimization design was carried out and the design process is shown in Fig.14. 


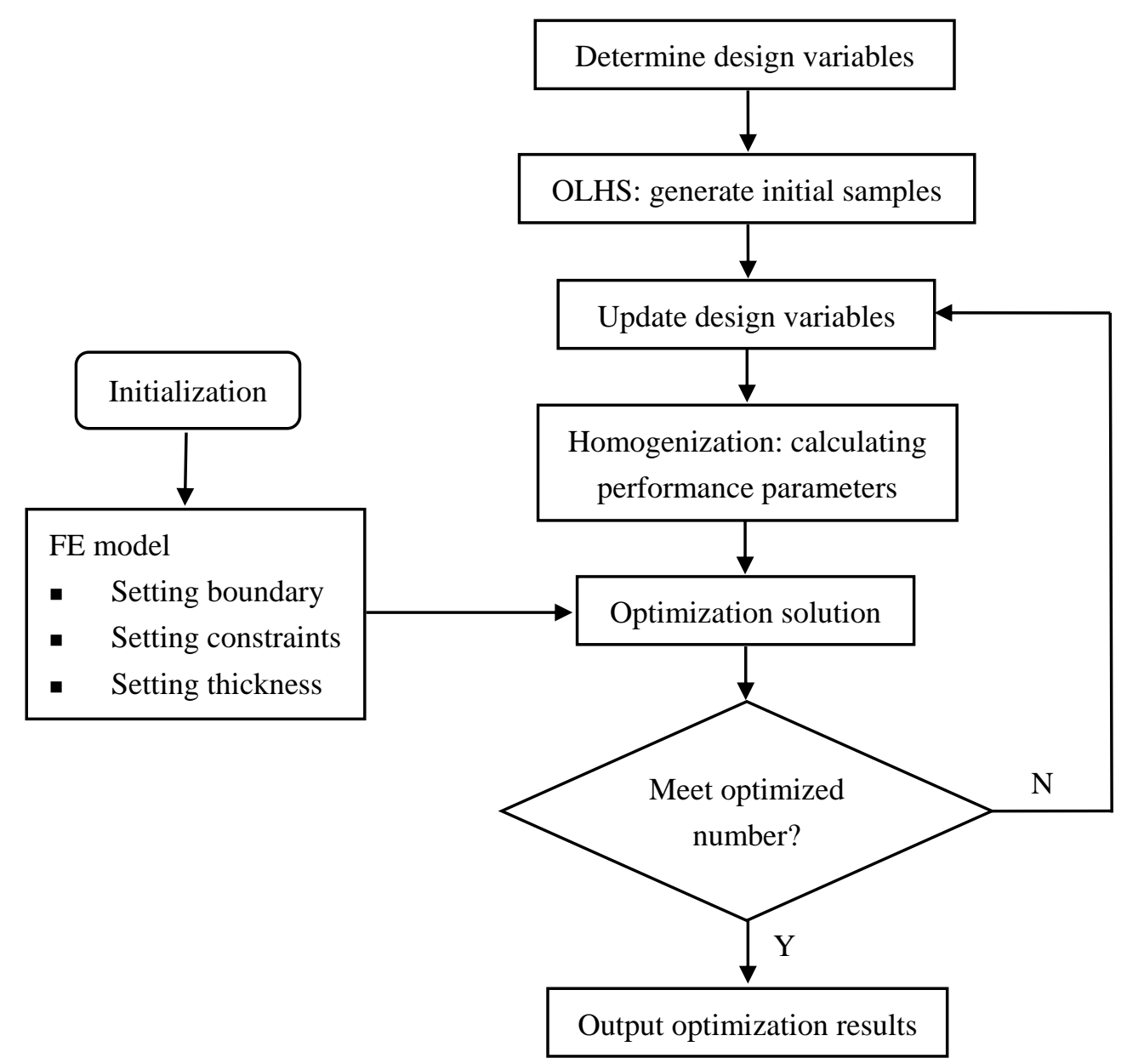

Fig. 14. Flowchart of optimization design process

\subsection{Numerical implementation}

The automobile hood is one of the important auto parts, which is currently formed mainly by stamping steel plates. It is usually a member made up of parts such as inner, outer plates and reinforcements. The fiber reinforced composites have become the choice for lightweight vehicles and have been favored by car companies all over the world. In this study, LCFNC was applied to the optimal design of engine cover, and the performance of carbon fiber composite engine cover was studied by finite element simulation.

The software of Hyperworks was utilized to model and assemble the hood. A quadrilateral shell element with a basic size of $10 \mathrm{~mm} \times 10 \mathrm{~mm}$ was adopted for the element, and the local refinement of the element was carried out for the key positions. Besides, considering the calculation time and 
accuracy of the model, the minimum element size is set to be greater than $3 \mathrm{~mm}$. Gauss integration is adopted for the integration of elements. The finite element model of automobile engine hood and some parts is shown in Fig. 15. The number of elements and nodes of the engine hood is 58456 and 62426, respectively.

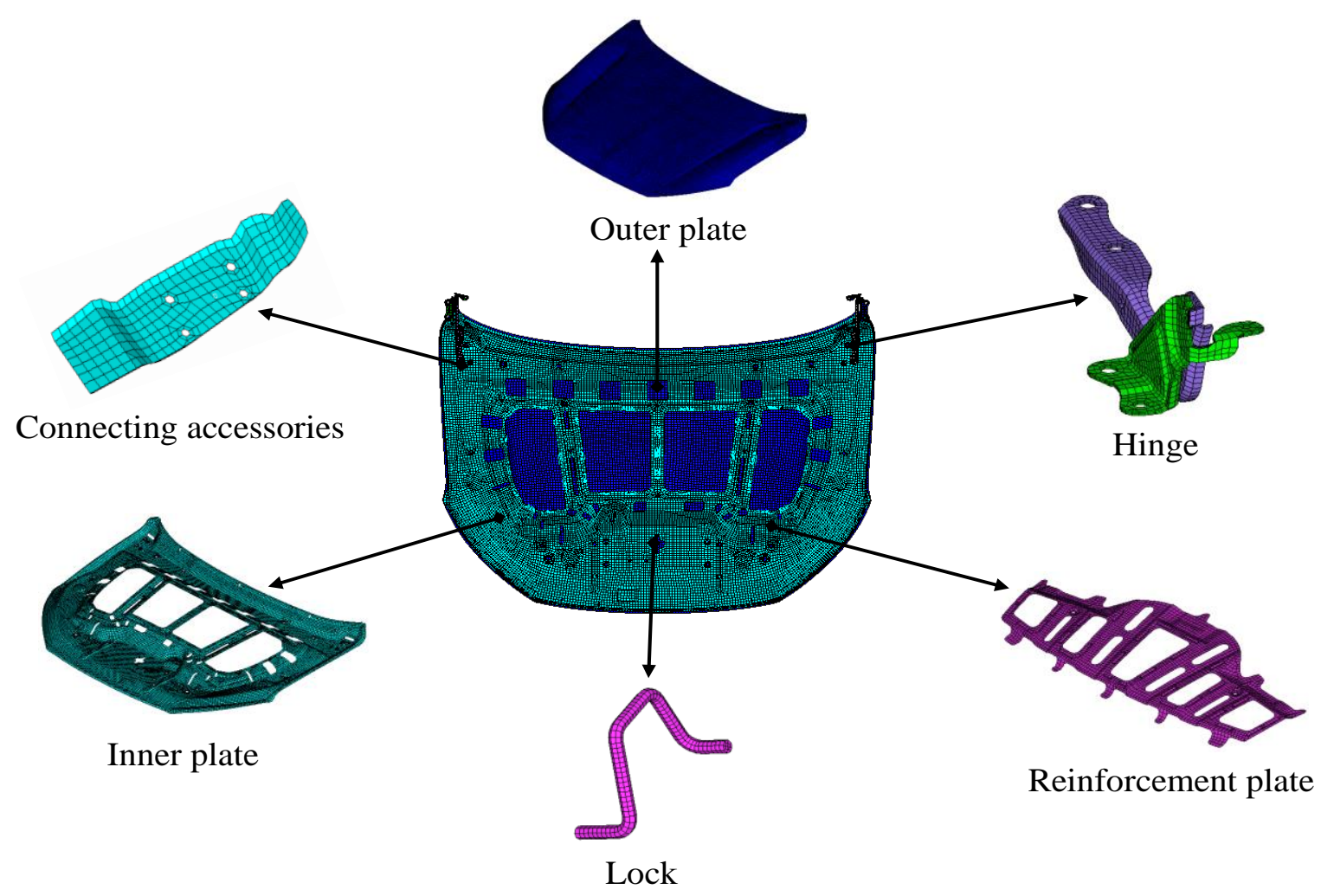

Fig. 15 The Finite element model of hood

In this work, the torsional stiffness, bending stiffness, lateral stiffness and first-order mode of the engine hood were analyzed by finite element method to meet the basic performance requirements of the engine hood. For different working conditions, loads and constraints need to be applied at corresponding nodes and regions. The boundary conditions for torsional stiffness analysis are shown in Fig 16 (a): a force of $100 \mathrm{~N}$ in the z-axis direction is applied to the $50 \mathrm{~mm} \times 50 \mathrm{~mm}$ region of the outer plate corresponding to the buffer block. RBE2 element was used to simulate the bolt, and all the degrees-of-freedom of the center node were fixed. Marks 1, 2 and 3 respectively indicate that the translation degrees of freedom along the $\mathrm{x}, \mathrm{y}$ and $\mathrm{z}$ directions are fixed. Marks 4, 5 and 6 respectively 
indicate that the rotation degrees of freedom along the $\mathrm{x}, \mathrm{y}$ and $\mathrm{z}$ directions are limited. For the analysis of bending stiffness and lateral stiffness, a force of $180 \mathrm{~N}$ in the $\mathrm{z}$-axis direction is applied to the front inflection point and a force of $196.2 \mathrm{~N}$ in the $\mathrm{z}$-axis direction is applied at the lock core node, respectively. The details of constraints and loads are shown in Fig 16(b)-(c). For the first-order modal analysis, no constraints and loads are imposed on the components. Through finite element analysis, the feasibility of replacing the steel parts with LCFNC parts was investigated and the lightweight effect was tested.

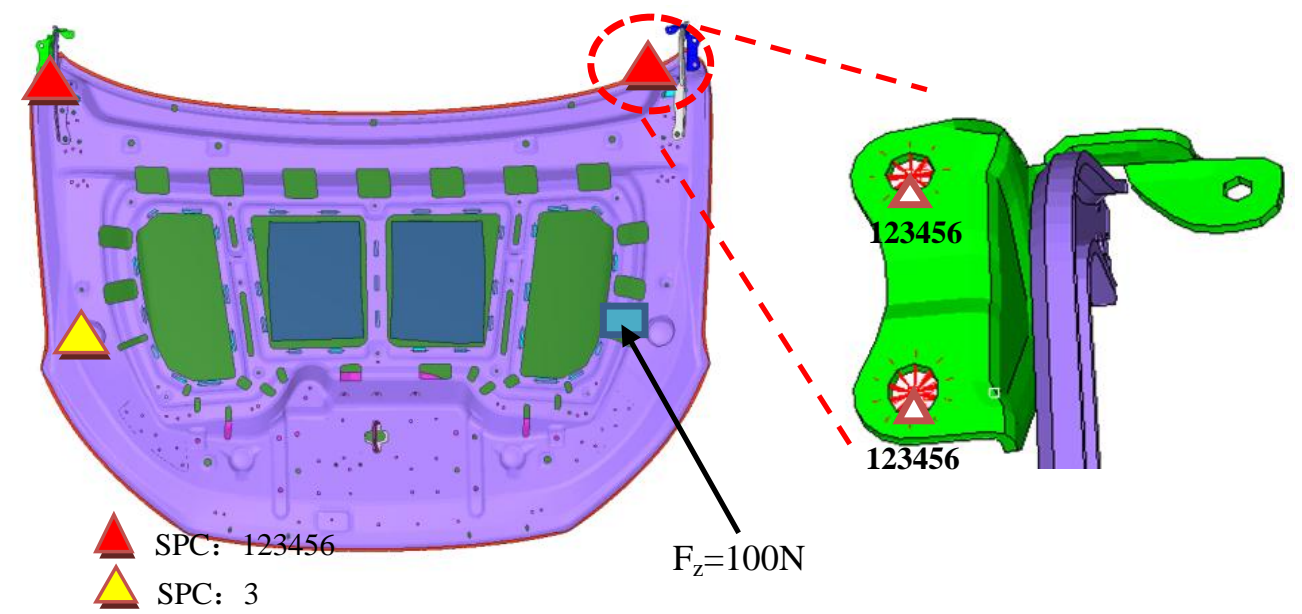

(a)

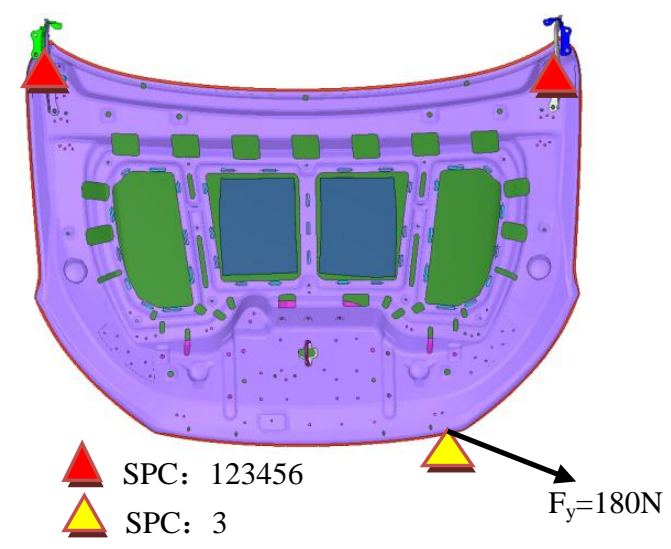

(b)

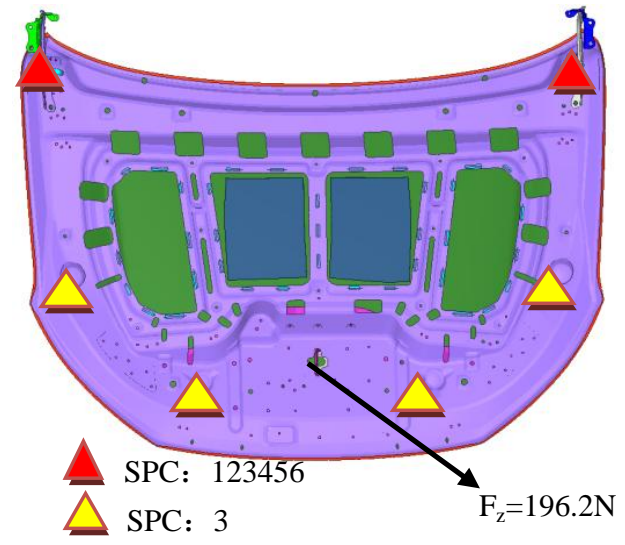

(c)

Fig. 16 Schematic diagram of constraints and loads under different analysis conditions (a) Torsional Stiffness, (b) Lateral stiffness, (c) bending stiffness

\subsection{Variables and Constraints}

In general, the main components of the engine cover are outer plate, inner plate and 
reinforcement plate, which are selected for the design field (Fig. 17). The volume fraction of composite and thickness of the components are defined as design variables.

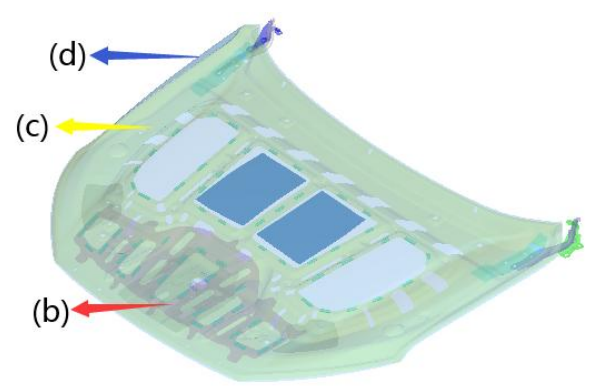

(a)

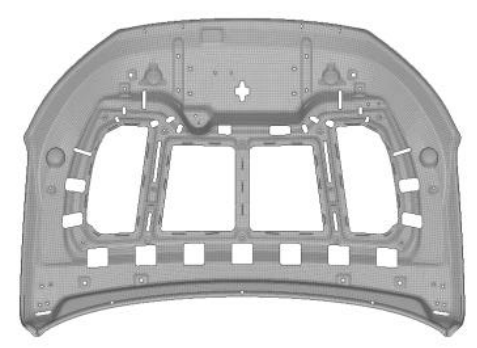

(c)

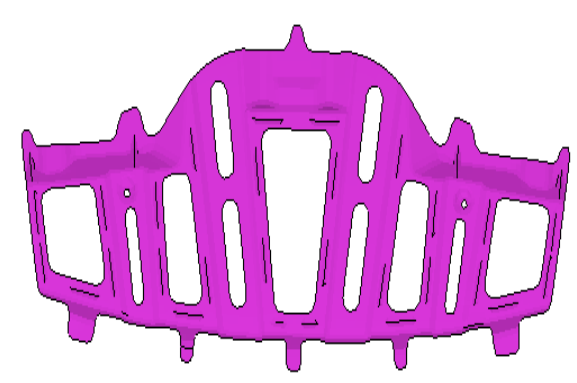

(b)

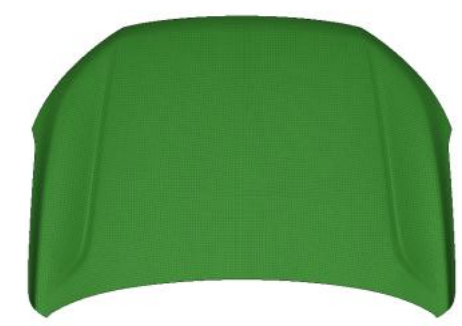

(d)

Fig. 17. The engine cover (a) and key components, reinforcement plate (b), outer plate(c), inner plate (d)

In the lightweight design of vehicles, the effective approach is to adopt new materials and new structures [44]. From these two perspectives, the substitution of materials can be achieved indirectly by changing micro-parameters. We set the volume fraction range from 0.2 to 0.4 . In this interval, the LCFNC has a good plane isotropy. The macro structure of the component is adjusted by thickness which is set as a continuous variable. The design variables are shown in Table 2.

Table2 design variables and value interval.

\begin{tabular}{|c|c|c|c|c|c|}
\hline Sign & Design variable & Type & Original value & Minimum & Maximum \\
\hline$V_{1}$ & Volume fraction of outer plate & discrete & l & 0.2 & 0.4 \\
\hline$V_{2}$ & Volume fraction of inner plate & discrete & / & 0.2 & 0.4 \\
\hline$V_{3}$ & Volume fraction of reinforcement & discrete & / & 0.2 & 0.4 \\
\hline$h_{1}$ & Thickness of outer plate & continuous & 0.7 & 1.0 & 2.0 \\
\hline$h_{2}$ & Thickness of inner plate & continuous & 0.65 & 1.0 & 2.0 \\
\hline
\end{tabular}


In the optimization design process, it is necessary to ensure the basic performance requirements of components, such as stiffness and hardness. According to the idea of the equivalent generation design, the original steel hood was adopted as the reference standard. The performance indexes are required not to be lower than the corresponding reference values. For the hood, the values of $N_{1}$, $N_{2}$ and $R$ are $150 \mathrm{~N} \cdot \mathrm{m} / \mathrm{deg}, 0.98 \mathrm{~mm}$ and 25 respectively. Besides, there are boundary constraints for the design variables, namely the thickness of components and the fiber volume fraction of material (Table 2).

\subsection{Results and discussion}

OLHS was employed to generate sample points containing different volume fractions and part thicknesses. 180 sample points were created to perform finite element analysis. The average calculation time of one cycle of optimization model is about 32 minutes. Non-dominated sorting genetic algorithm (NSGA-II) were applied to search the optimal solution and the relevant parameters of the algorithm are shown in Table 3. Depending on the optimization model, the multidisciplinary optimization software isight was used for deterministic optimization in this work.

Table3 The parameters in the non-dominated sorting genetic algorithm

\begin{tabular}{cc}
\hline Parameter & Value \\
\hline Population size & 24 \\
Number of generations & 30 \\
Crossover probability & 0.9 \\
Crossover distribution index & 15 \\
Mutation distribution index & 20 \\
\hline
\end{tabular}

The number of iteration during optimization process is 720 , and the number of the feasible solutions within the design objectives is 680 . The optimization results are shown in Table 4 Table 4 Comparison of initial and optimization parameters

$\begin{array}{lll}\text { Sign } & \text { Original value } & \text { Optimization value }\end{array}$




\begin{tabular}{cccc}
\hline & $V_{1}$ & $/$ & 0.38 \\
Design variable & $V_{2}$ & $/$ & 0.36 \\
& $V_{3}$ & $/$ & 0.38 \\
& $h_{1}$ & 0.7 & 1.8 \\
& $h_{2}$ & 0.65 & 1.6 \\
& $h_{3}$ & 0.7 & 1.0 \\
\hline \multirow{2}{*}{ Constraint } & $\kappa$ & 26 & 30 \\
condition & $c_{1}$ & 270 & 195 \\
& $u_{2}$ & 0.75 & 0.74 \\
\hline Objective & $u_{1}$ & 0.97 & 0.88 \\
output & $m$ & 20.58 & 12.94 \\
\hline
\end{tabular}

As shown in Table 4, the optimized carbon fiber hood is $7.64 \mathrm{~kg}$ lighter, reducing the overall weight by $37 \%$. The lightweight effect is obvious, which plays an important role in effectively reducing fuel consumption and reducing emissions without compromising the vehicle safety. At the same time, the displacement of optimized lateral stiffness is 0.88 , which means the later stiffness is $9 \%$ higher than the original value. For other mechanical properties, both torsional stiffness and first-order modal performance have been improved, while bending stiffness performance has been reduced but still within the safe range. The performance comparison before and after optimization of some working conditions is shown in Fig 18.



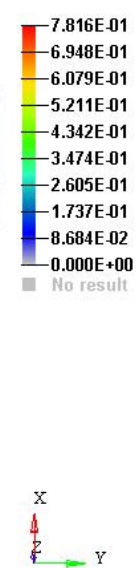

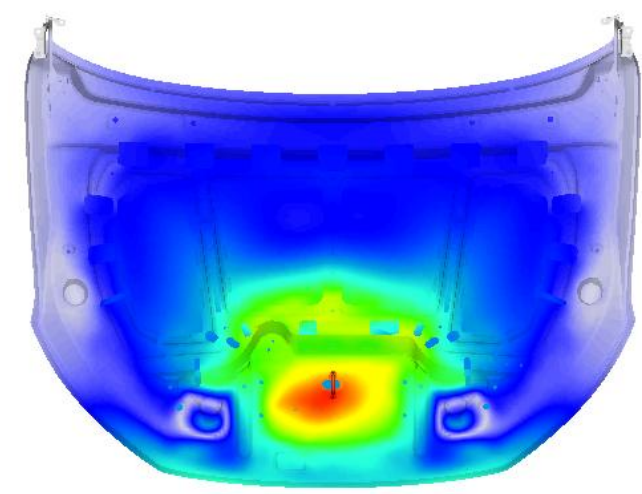

(a) 

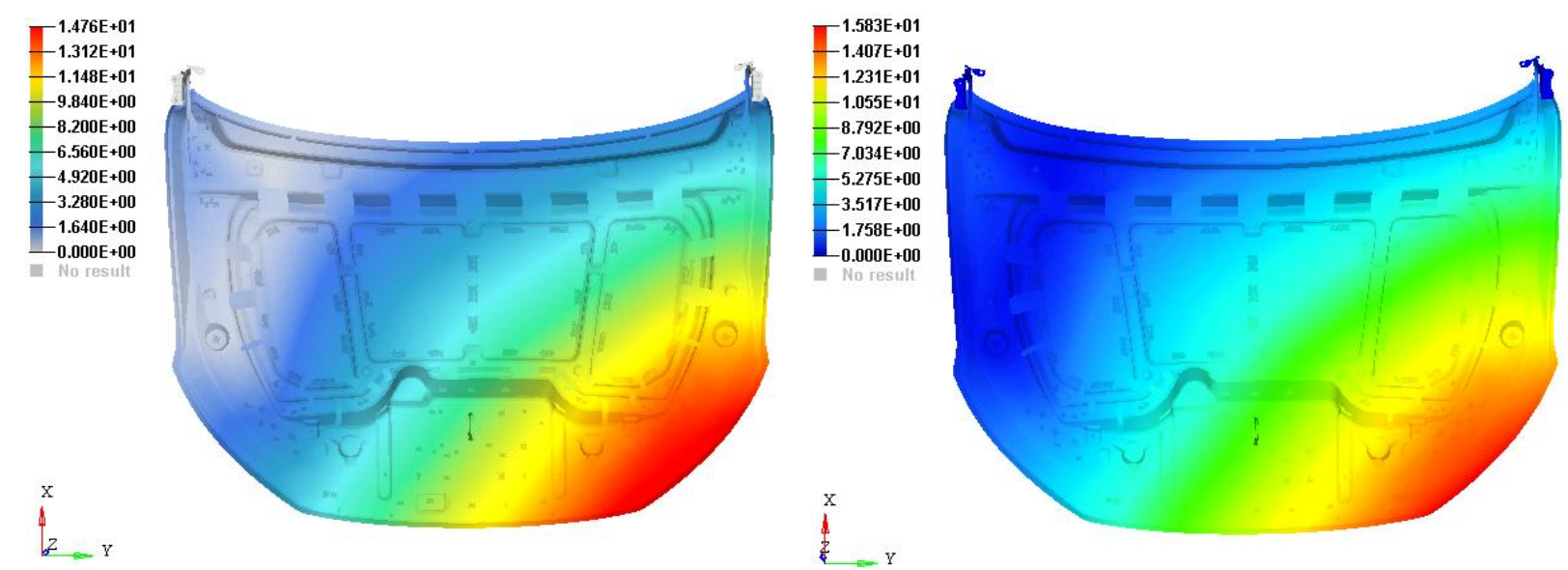

(b)
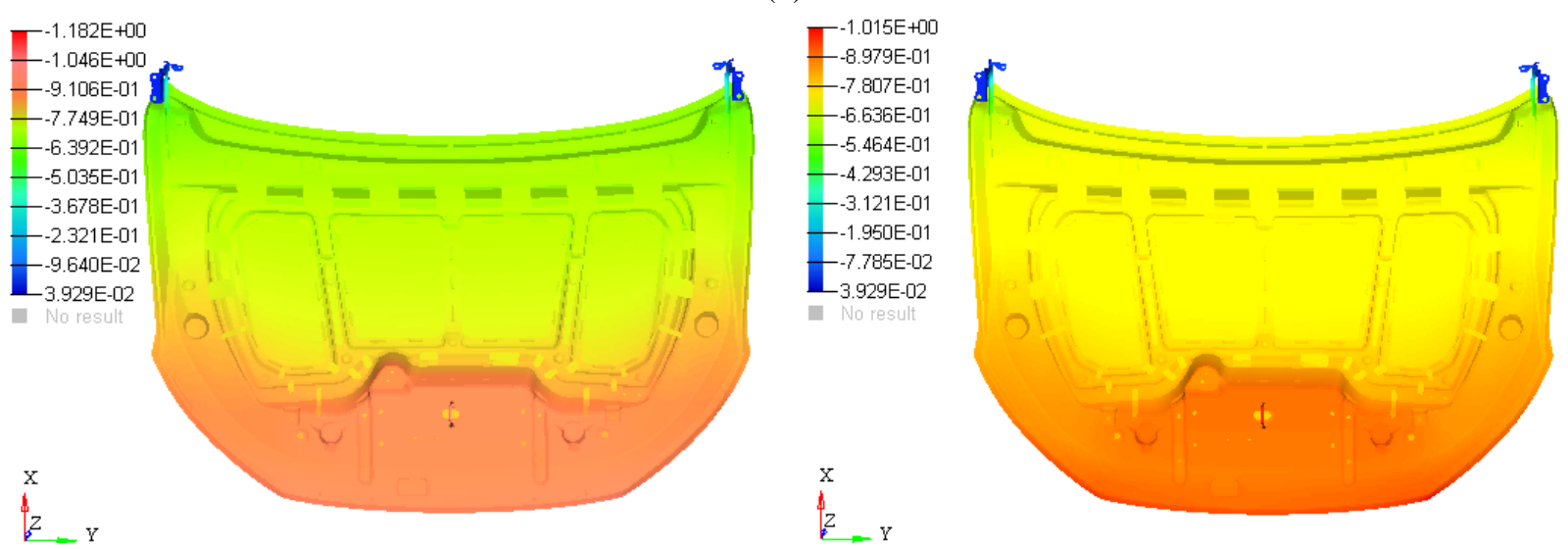

(c)

Fig. 18. Comparison of mechanical properties between engine made with composite(right) and engine made with steel(left) : bending stiffness (a), torsional stiffness (b) and lateral stiffness (c)

Generally speaking, by optimizing the micro-material parameters and thickness of the carbon fiber hood, the lightweight can be achieved while improving performance on the premise of the safety. The advantages are very obvious.

\section{Conclusions}

Aiming at weakening the application obstacles of carbon fibers caused by high cost and manufacturing process, a novel plane isotropic composite reinforced by long carbon fiber nonwoven is introduced. The tensile properties of material were studied experimentally and analytically. Analytical results predicted by homogenization method show considerable agreement with those generated from the experiments within the volume fraction range of 0.2-0.4. The lower or higher 
volume fraction makes the properties of LCFNC tend to be unstable and weakened. The effects of the aspect ratio of fibers on the tensile properties of materials were investigated in detail, and a critical value was found based on the predictions.

Considering the coupling of microscopic parameters and macroscopic dimensions of materials, a multi-scale optimization method is proposed to achieve lightweight design and maximum mechanical properties of composites. Finally, the long carbon fiber non-woven composite (LCFNC) and multi-scale optimization method were applied to the multi-objective optimization design of engine hood. The finite element simulations show that the weight of composite hood was reduced by $37 \%$, while exhibiting better later stiffness and torsional stiffness in comparison with the conventional steel. The bending stiffness performance has been reduced but still within the safe range.

\section{Acknowledgement}

The project is supported by the Foundation for Innovative Research Groups of the Natural Science Foundation of China (Grant No. U1864207) and the National Natural Science Foundation of China (Grant No. 51621004), the Opening Project of the Guangxi Key Laboratory of Automobile Components and Vehicle Technology of Guangxi University of Science and Technology (No. 2017GKLACVTKF01) and Guangxi Science and Technology Project (No. 2017AA10104).

\section{Data availability}

The raw data and processed data required to reproduce these findings cannot be shared at this time as the data also forms part of an ongoing study. 


\section{References}

[1] Fuchs ERH, Field FR, Roth R, Kirchain RE. Strategic materials selection in the automobile body: Economic opportunities for polymer composite design. Composites Science \& Technology. 2008;68:1989-2002.

[2] Becke J, Stieg J. Advanced SMC-Material concepts for lightweight exterior vehicle surfaces. European Alliance for SMC, Automotive Seminar, Bremen, Germany February2004.

[3] Das S. The cost of automotive polymer composites: a review and assessment of DOE's lightweight materials composites research: Oak Ridge National Laboratory Oak Ridge, Tennessee, USA, 2001.

[4] Mubashir S, Muttana SB. Carbon fibre composites: Outlook for the automobile sector. Auto Tech Review. 2012;1:18-23.

[5] Cramer DR, Taggart DF, Inc H. Design and manufacture of an affordable advanced-composite automotive body structure. Proceedings from The 19th international battery, hybrid and fuel cell electric vehicle symposium and exhibition2002. p. 1-12.

[6] Thilagavathi G, Pradeep E, Kannaian T, Sasikala L. Development of natural fiber nonwovens for application as car interiors for noise control. Journal of Industrial Textiles. 2010;39:267-78.

[7] Lin X, Zhu H, Yuan X, Wang Z, Bordas S. The elastic properties of composites reinforced by a transversely isotropic random fibre-network. Composite structures. 2019;208:33-44.

[8] Efendy MA, Pickering K. Comparison of strength and Young modulus of aligned discontinuous fibre PLA composites obtained experimentally and from theoretical prediction models. Composite structures. 2019;208:566-73.

[9] Eshelby JD. The determination of the elastic field of an ellipsoidal inclusion, and related problems. Proceedings of the Royal Society of London Series A Mathematical and Physical Sciences. 1957;241:376-96.

[10] Mori T, Tanaka K. Average stress in matrix and average elastic energy of materials with misfitting inclusions. Acta metallurgica. 1973;21:571-4.

[11] Christensen R, Waals F. Effective stiffness of randomly oriented fibre composites. Journal of Composite Materials. 1972;6:518-32.

[12] Cox H. The elasticity and strength of paper and other fibrous materials. British journal of applied physics. 1952;3:72.

[13] Hashimoto M, Okabe T, Sasayama T, Matsutani H, Nishikawa M. Prediction of tensile strength of discontinuous carbon fiber/polypropylene composite with fiber orientation distribution. Composites Part A: Applied Science and Manufacturing. 2012;43:1791-9.

[14] Hartl A, Jerabek M, Freudenthaler P, Lang R. Orientation-dependent compression/tension asymmetry of short glass fiber reinforced polypropylene: Deformation, damage and failure. Composites Part A: Applied Science and Manufacturing. 2015;79:14-22.

[15] Giordano S. Order and disorder in heterogeneous material microstructure: Electric and elastic characterisation of dispersions of pseudo-oriented spheroids. International journal of engineering science. 2005;43:1033-58.

[16] Berkache K, Deogekar S, Goda I, Picu R, Ganghoffer J-F. Construction of second gradient continuum models for random fibrous networks and analysis of size effects. Composite Structures. 2017;181:347-57.

[17] Delincé M, Delannay F. Elastic anisotropy of a transversely isotropic random network of interconnected fibres: non-triangulated network model. Acta Materialia. 2004;52:1013-22.

[18] Ma Y, Zhu H, Su B, Hu G, Perks R. The elasto-plastic behaviour of three-dimensional stochastic fibre networks with cross-linkers. Journal of the Mechanics and Physics of Solids. 2018;110:155-72.

[19] Benveniste Y. A new approach to the application of Mori-Tanaka's theory in composite materials. Mechanics of materials. 1987;6:147-57.

[20] Kc B, Faruk O, Agnelli J, Leao A, Tjong J, Sain M. Sisal-glass fiber hybrid biocomposite: Optimization of injection 
molding parameters using Taguchi method for reducing shrinkage. Composites Part A: Applied Science and Manufacturing. 2016;83:152-9.

[21] Ghiasi H, Fayazbakhsh K, Pasini D, Lessard L. Optimum stacking sequence design of composite materials Part II: Variable stiffness design. Composite Structures. 2010;93:1-13.

[22] Ghiasi H, Pasini D, Lessard L. Optimum stacking sequence design of composite materials Part I: Constant stiffness design. Composite Structures. 2009;90:1-11.

[23] Blasques JP, Stolpe M. Maximum stiffness and minimum weight optimization of laminated composite beams using continuous fiber angles. Structural and Multidisciplinary Optimization. 2011;43:573-88.

[24] Huang X, Zhou S, Xie Y, Li Q. Topology optimization of microstructures of cellular materials and composites for macrostructures. Computational Materials Science. 2013;67:397-407.

[25] Belingardi G, Beyene AT, Koricho EG. Geometrical optimization of bumper beam profile made of pultruded composite by numerical simulation. Composite Structures. 2013;102:217-25.

[26] Bruyneel M. A general and effective approach for the optimal design of fiber reinforced composite structures. Composites Science \& Technology. 2006;66:1303-14.

[27] Lee H, Choi C-w, Jin J-w, Huh M, Lee S, Kang K-w. Homogenization-based multiscale analysis for equivalent mechanical properties of nonwoven carbon-fiber fabric composites. Journal of Mechanical Science and Technology. 2019;33:4761-70.

[28] Pan Y, Iorga L, Pelegri AA. Numerical generation of a random chopped fiber composite RVE and its elastic properties. Composites Science and Technology. 2008;68:2792-8.

[29] Agarwal BD, Broutman LJ, Chandrashekhara K. Analysis and performance of fiber composites: John Wiley \& Sons, 2006.

[30] Abaimov SG, Trofimov A, Sergeichev IV, Akhatov IS. Multi-step homogenization in the Mori-Tanaka-Benveniste theory. Composite structures. 2019;223:110801.

[31] Hill R. Elastic properties of reinforced solids: Some theoretical principles. Journal of the Mechanics \& Physics of Solids. 1963;11:357-72.

[32] Eshelby JD. The Determination of the Elastic Field of an Ellipsoidal Inclusion, and Related Problems. Proceedings of the Royal Society of London. 1957;241:376-96.

[33] Ren C, Chen J, Pan H. Prediction model for elastic modulus of random short fiber reinforced composite. Acta Materiae Compositae Sinica. 2012;29:191-4.

[34] Doghri I, Chandrasekharaiah DS. Mechanics of Deformable Solids: Linear and Nonlinear, Analytical and Computational Aspects. Applied Mechanics Reviews. 2001;54:83.

[35] Nguyen VD, Béchet E, Geuzaine C, Noels L. Imposing periodic boundary condition on arbitrary meshes by polynomial interpolation. Computational Materials Science. 2012;55:390-406.

[36] Hu Y, Zhang Y, Fu X, Hao G, Jiang W. Mechanical properties of Ti/CF/PMR polyimide fiber metal laminates with various layup configurations. Composite structures. 2019;229:111408.

[37] Bisaria H, Gupta M, Shandilya Pa, Srivastava R. Effect of fibre length on mechanical properties of randomly oriented short jute fibre reinforced epoxy composite. Materials Today: Proceedings. 2015;2:1193-9.

[38] Kumar R, Kumar K, Sahoo P, Bhowmik S. Study of mechanical properties of wood dust reinforced epoxy composite. Procedia Materials Science. 2014;6:551-6.

[39] Hou S, Han XU, Sun G, Long S, Wei LI, Yang X, et al. Multiobjective optimization for tapered circular tubes. Thin-Walled Structures. 2011;49:855-63.

[40] Pholdee N, Bureerat S. An efficient optimum Latin hypercube sampling technique based on sequencing optimisation using simulated annealing. International Journal of Systems Science. 2015;46:1780-9.

[41] Deb K, Agrawal S, Pratap A, Meyarivan T. A fast elitist non-dominated sorting genetic algorithm for multi-objective 
optimization: NSGA-II. International conference on parallel problem solving from nature: Springer; 2000. p. 849-58.

[42] Vo-Duy T, Duong-Gia D, Ho-Huu V, Vu-Do HC, Nguyen-Thoi T. Multi-objective optimization of laminated composite beam structures using NSGA-II algorithm. Composite Structures.168:498-509.

[43] Murugan P, Kannan S, Baskar S. NSGA-II algorithm for multi-objective generation expansion planning problem. Electric Power Systems Research.79:622-8.

[44] Duan L, Sun G, Cui J, Tao C, Cheng A, Li G. Crashworthiness design of vehicle structure with tailor rolled blank. Structural \& Multidisciplinary Optimization. 2016;53:321-38. 